OPEN ACCESS

Edited by:

Hong-Bo Zhao,

University of Kentucky, USA

Reviewed by:

Chiara Donati,

University of Florence, Italy Esperanza Bas Infante,

University of Miami, USA

*Correspondence:

Renjie Chai

renjiec@seu.edu.cn

Huawei L

lihuawei63@gmail.com

Haibo Shi

haibo99@hotmail.com

tThese authors have contributed equally to this work.

Received: 25 February 2017 Accepted: 11 April 2017

Published: 26 April 2017

Citation:

Cheng C, Guo L, LU L, XU X, Zhang $S$, Gao J, Waqas $M$, Zhu $C$, Chen Y, Zhang X, Xuan C, Gao X,

Tang M, Chen F, Shi H, Li H and Chai $R$ (2017) Characterization of the Transcriptomes of Lgr5+ Hair Cell Progenitors and Lgr5- Supporting

Cells in the Mouse Cochlea.

Front. Mol. Neurosci. 10:122. doi: 10.3389/fnmol.2017.00122

\section{Characterization of the Transcriptomes of Lgr5+ Hair Cell Progenitors and Lgr5- Supporting Cells in the Mouse Cochlea}

Cheng Cheng ${ }^{1,2,3+}$, Luo Guo ${ }^{4,5+}$, Ling Lu ${ }^{6,7+}$, Xiaochen Xu${ }^{1}$, ShaSha Zhang ${ }^{1}$, Junyan Gao ${ }^{8}$, Muhammad Waqas ${ }^{1,9}$, Chengwen Zhu ${ }^{6}$, Yan Chen ${ }^{4,5}$, Xiaoli Zhang ${ }^{6}$, Chuanying Xuan ${ }^{1}$, Xia Gao ${ }^{6}$, Mingliang Tang ${ }^{1}$, Fangyi Chen ${ }^{10}$, Haibo Shi ${ }^{11 *}$, Huawei Li $^{4,5,12,13 *}$ and Renjie Chai ${ }^{1,2,3 *}$

\begin{abstract}
1 Key Laboratory for Developmental Genes and Human Disease, Ministry of Education, Institute of Life Sciences, Southeast University, Nanjing, China, ${ }^{2}$ Research Institute of Otolaryngology, Nanjing, China, ${ }^{3}$ Co-innovation Center of Neuroregeneration, Nantong University, Nantong, China, ${ }^{4}$ Department of Otorhinolaryngology, Affiliated Eye and ENT Hospital, State Key Laboratory of Medical Neurobiology, Fudan University, Shanghai, China, ${ }^{5}$ Key Laboratory of Hearing Medicine of National Health and Family Planning Commission, Shanghai, China, ${ }^{6}$ Department of Otolaryngology-Head and Neck Surgery, Nanjing Drum Tower Hospital, Nanjing University Medical School, Nanjing, China, ${ }^{7}$ Department of Otolaryngology-Head and Neck Surgery, Drum Tower Clinical Medical College of Nanjing Medical University, Nanjing, China, ${ }^{8}$ Health Management and Policy, College of Public Health, Saint Louis University, St. Louis, MO, USA, ${ }^{9}$ Department of Biotechnology, Federal Urdu University of Arts, Science and Technology, Gulshan-e-lqbal, Pakistan, ${ }^{10}$ Department of Biomedical Engineering, Southern University of Science and Technology, Shenzhen, China, "1 Department of Otorhinolaryngology Head and Neck Surgery, The Sixth People's Hospital, Shanghai Jiao Tong University, Shanghai, China, ${ }^{12}$ Institutes of Biomedical Sciences, Fudan University, Shanghai, China, ${ }^{13}$ Shanghai Engineering Research Centre of Cochlear Implant, Shanghai, China
\end{abstract}

Cochlear supporting cells (SCs) have been shown to be a promising resource for hair cell $(\mathrm{HC})$ regeneration in the neonatal mouse cochlea. Previous studies have reported that Lgr5+ SCs can regenerate HCs both in vitro and in vivo and thus are considered to be inner ear progenitor cells. Lgr5+ progenitors are able to regenerate more HCs than Lgr5- SCs, and it is important to understand the mechanism behind the proliferation and $\mathrm{HC}$ regeneration of these progenitors. Here, we isolated Lgr5+ progenitors and Lgr5- SCs from Lgr5-EGFP-CreERT2/Sox2-CreERT2/Rosa26-tdTomato mice via flow cytometry. As expected, we found that Lgr5+ progenitors had significantly higher proliferation and HC regeneration ability than Lgr5- SCs. Next, we performed RNASeq to determine the gene expression profiles of Lgr5+ progenitors and Lgr5- SCs. We analyzed the genes that were enriched and differentially expressed in Lgr5+ progenitors and Lgr5-SCs, and we found 8 cell cycle genes, 9 transcription factors, and 24 cell signaling pathway genes that were uniquely expressed in one population but not the other. Last, we made a protein-protein interaction network to further analyze the role of these differentially expressed genes. In conclusion, we present a set of genes that might regulate the proliferation and $\mathrm{HC}$ regeneration ability of Lgr5+ progenitors, and these might serve as potential new therapeutic targets for $\mathrm{HC}$ regeneration. 


\section{INTRODUCTION}

Sensorineural hearing loss is a common sensory disorder caused by the loss of hair cells (HCs). HCs are responsible for converting vibrational sound waves into the electrical impulses that are transmitted to the brain. Regeneration of damaged HCs could possibly yield a cure for sensorineural hearing loss, which still has no treatment other than prosthetic devices. Although the mature mammalian cochlea lacks the ability to regenerate $\mathrm{HCs}$, new $\mathrm{HCs}$ are spontaneously regenerated in non-mammalian vertebrates from the resident supporting cells (SCs) that surround the HCs (Corwin and Cotanche, 1988; Balak et al., 1990; Roberson et al., 1992; Stone and Cotanche, 2007; Bermingham-McDonogh and Reh, 2011; Warchol, 2011). Previous studies have shown that some cochlear SCs in neonatal mammals are HC progenitors that possess a limited capacity to regenerate HCs (Chai et al., 2012; Shi et al., 2013; Bramhall et al., 2014; Cox et al., 2014; Wang et al., 2015). However, these SCs lose their intrinsic regenerative potential as the animal ages (Oesterle et al., 2008; Bermingham-McDonogh and Reh, 2011; Warchol, 2011; Cox et al., 2014), and as a result hearing loss tends to be permanent and incurable in mature mammals.

Supporting cells in the mouse inner ear are known to be a potential resource for HC regeneration after damage (White et al., 2006; Sinkkonen et al., 2011), and when isolated by flow cytometry neonatal SCs have the ability to proliferate and to differentiate into HCs in vitro. Upon HC injury, cochlear SCs also display a limited capacity to proliferate and to regenerate HCs (White et al., 2006; Sinkkonen et al., 2011). Leucine-rich repeat-containing G-protein coupled receptor 5 (Lgr5), a Wnt target gene, is a marker of endogenous stem cells in rapidly proliferating organs (Barker et al., 2007; Jaks et al., 2008). Lgr5 is widely expressed in the cochlear duct prosensory region during embryonic development, but it becomes restricted to a subset of SCs after birth (Chai et al., 2011). Recently, Lgr5+ cells in newborn mice have been shown to be a population of HC progenitors that can regenerate HCs through both direct differentiation and through mitotic regeneration (Chai et al., 2012; Shi et al., 2013; Bramhall et al., 2014; Cox et al., 2014; Hegarty et al., 2015; Wang et al., 2015; Lu et al., 2016). However, the detailed gene expression profile differences between the Lgr5+ progenitors and the Lgr5- SCs have not yet been investigated.

The current study focused on identifying the molecular mechanism behind the increased proliferation and HC regeneration ability of Lgr5+ progenitors in the neonatal mouse cochlea. We performed RNA-Seq profiling of the Lgr5+ progenitors and the Lgr5- SCs from Lgr5-EGFP-CreERT2/Sox2CreERT2/Rosa26-tdTomato transgenic mice and identified the differentially expressed genes that might be involved in regulating proliferation, differentiation, or signaling pathways in these two cell populations. To further analyze the role of differentially expressed genes between Lgr5+ progenitors and Lgr5- SCs, we created a protein-protein interaction network using STRING (Search Tool for the Retrieval of Interacting Genes/Proteins). These datasets are expected to serve as a resource for future work in determining the detailed regulatory mechanisms behind HC regeneration.

\section{MATERIALS AND METHODS}

\section{Mice and Genotyping}

Lgr5 EGFP-Ires-CreERT2 (stock no. 008875) (Barker et al., 2007), Sox2-CreER (stock no. 008875), and Rosa26-tdTomato (stock no. 007914) (Madisen et al., 2010) mice were obtained from the Jackson Laboratory. Transgenic mice were genotyped using genomic DNA from tail tips by adding $180 \mu 150 \mathrm{mM} \mathrm{NaOH}$, incubating at $98^{\circ} \mathrm{C}$ for $1 \mathrm{~h}$, and adding $20 \mu \mathrm{l} \mathrm{M}$ Tris-HCl. The genotyping primers were as follows: Lgr5: (F) CTG CTC TCT GCT CCC AGT CT; wild-type (R) ATA CCC CAT CCC TTT TGA GC; mutant (R) GAA CTT CAG GGT CAG CTT GC; tdTomato: wild-type (F) AAG GGA GCT GCA GTG GAG T; (R) CCG AAA ATC TGT GGG AAG TC; mutant (F) GGC ATT AAA GCA GCG TAT C; (R) CTG TTC CTG TAC GGC ATG G. Sox2-CreER mutant (F) GCG GTC TGG CAG TAA AAA CTA TC; Sox2-CreER mutant (R) GTG AAA CAG CAT TGC TGT CAC TT; Sox2-CreER wild-type (F) CTA GGC CAC AGA ATT GAA AGA TCT; Sox2-CreER wild-type (R) GTA GGT GGA AAT TCT AGC ATC ATC C. Tamoxifen (Sigma, diluted in corn oil) was injected i.p. at post-natal day (P) 2 at $0.1 \mathrm{mg} / \mathrm{g}$ bodyweight to induce the Cre recombinase activity, and the cochleae were harvested at P4. All animal procedures were performed according to protocols approved by the Animal Care and Use Committee of Southeast University and were consistent with the National Institutes of Health Guide for the Care and Use of Laboratory Animals. All efforts were made to minimize the number of animals used and to prevent their suffering.

\section{Immunostaining}

The dissected cochleae were fixed in $4 \%$ paraformaldehyde for $1 \mathrm{~h}$ at room temperature, washed three times with $1 \times$ PBST (0.1\% Triton X-100 in PBS), and incubated for $1 \mathrm{~h}$ at room temperature in blocking medium (1\% Triton X-100, 1\% BSA, $10 \%$ heat-inactivated donkey serum, and $0.02 \%$ sodium azide in PBS at pH 7.2). The primary antibody was diluted in PBT1 (10\% Triton X-100, 1\% BSA, 5\% heat-inactivated goat serum, and $0.02 \%$ sodium azide in PBS at $\mathrm{pH} 7.2$ ) and incubated with the samples overnight at $4^{\circ} \mathrm{C}$. The samples were washed three times with $1 \times$ PBST, and the secondary antibody [diluted in PBT2 (0.1\% Triton X-100 and 1\% BSA in PBS at pH 7.2)] was added for $1 \mathrm{~h}$ at room temperature. The samples were washed again three times with $1 \times$ PBST and then mounted on slides in DAKO. Cells were imaged with an LSM 700 confocal microscope. The antibodies used in this paper were anti-myosin7a (Proteus Bioscience, \#25-6790, 1:1000 dilution), anti-sox2 (Santa Cruz, \#sc-17320, 1:500 dilution), anti-myosin6 (Proteus Biosciences, \#25-6791, 1:500 dilution), anti-parvalbumin (Sigma, \#P3088), FM1-43 dye (Invitrogen, \# F35355), anti-espin1 (Transduction Labs, 1:200 dilution), Alexa Fluor ${ }^{\circledR} 647$ donkey anti-goat IgG $\left(\mathrm{H}+\mathrm{L}\right.$; Invitrogen, A-21447, 1:500 dilution), and Alexa Fluor ${ }^{\circledR}$ 555 donkey anti-rabbit IgG (H+L; Invitrogen, A-31572, 1:500 
dilution). Cell proliferation was measured with the Click-it EdU imaging kit (Invitrogen).

\section{Flow Cytometry}

We used the Lgr5-EGFP-CreERT2/Sox2-CreERT2/Rosa26tdTomato transgenic mice to isolate the Lgr5+ HC progenitors and the Lgr5- SCs. Tamoxifen (Sigma, diluted in corn oil) was injected at P2, and the mice were sacrificed at P4. The cochleae were dissected in cold $1 \times$ HBSS (Gibco) and transferred to $50 \mu \mathrm{l} 1 \times$ PBS in $1.5 \mathrm{ml}$ Eppendorf tubes, and the tissues were incubated in $50 \mu 10.25 \%$ trypsin-EDTA (Invitrogen; \#25200-056) for $8 \mathrm{~min}$ at $37^{\circ} \mathrm{C}$. The digestion was stopped by the addition of $50 \mu \mathrm{l}$ trypsin inhibitor (Worthington Biochem, \#LS003570), and $200 \mu \mathrm{l}$ blunt tips (Eppendorf, \#22491245) were used to triturate the tissue into single cell suspensions. The cells were filtered through a $40 \mu \mathrm{l}$ strainer (BD Biosciences, 21008-949) to eliminate clumps, and the EGFP+ cells were sorted on a BD FACS Aria III (BD Biosciences).

\section{Quantitative Real-Time PCR}

After FACS sorting, the Cells-to-cDNA II kit (Ambion, AM 1722) was used to extract total RNA from the collected cells and to reverse transcribe it into cDNA using oligo $(\mathrm{dT})$ primers. The SYBR Green PCR Master Mix (Roche) was used on a BIO-RAD C1000 Touch thermal cycler (BIO-RAD). Expression levels of Lgr5, Sox 2 , and Brn3.1 were normalized to the $\beta$-actin in the same samples. The primers were as follows:

Lgr5 (F) 5'-TCT TCA CCT CCT ACC TGG ACC T-3'; Lgr5 (R) 5'-GGC GTA GTC TGC TAT GTG GTG T-3'; Sox2 (F) 5'-ATG AAC GGC TGG AGC AAC GGC A-3'; Sox2 (R) 5'-TCA CAT GTG CGA CAG GGG CAG T-3'; Brn3.1 (F) 5'-ACC CAA ATT CTC CAG CCT ACA C-3'; Brn3.1 (R) $5^{\prime}$ GGC GAG ATG TGC TCA AGT AAG T-3'; E2f1(F) $5^{\prime}$-TCA CTA AAT CTG ACC ACC AAA CG-3'; E2f1(R) 5'-TTG GAC TTC TTG GCA ATG AGC-3'; Rad51(F) 5'-GTC CAC AGC CTA TTT CAC G-3'; Rad51(R) 5'-GCA TAA GCA ACA GCC TCC-3'; Aurka(F) 5'-CTT TCC CTG ACT TTG TGA C-3'; Aurka(R) 5'-CAG TGT TTC AGT CCC TTT C-3'; Ccnf(F) 5'-AGC ACA AAG CCT TGC CAC CAT C-3'; Ccnf(R) 5'AAG CCA GGT GCG TGT CCT TGT C-3'; Cdkn3(F) 5'GGA CTC CTG ACA TAG CCA GC-3'; Cdkn3(R) $5^{\prime}$-CTG TAT TGC CCC GGA TCC TC-3'; Trp63(F) 5'-TGG CAT TAG CCA TAG GTA GGC ACA-3'; Trp63(R) 5'-TCA CCA CCA AGT GAA GGA ATC CCA-3'; Barhl1(F) 5'-GGT ACC AGA ACC GCA GGA-3'; Barhl1(R) 5'-TGG AGC GCC GAG TAA TTG3'; Lbx2(F) 5'-GAG CGG CGA TTC GTC TTC-3'; Lbx2(R) $5^{\prime}$-TGT CTG GCA GTG CTG GGT A-3'; Nhlh1(F) $5^{\prime}$-GAC TCC AGT TCT GGA CTA AGT AAG-3'; Nhlh1(R) 5'-GGA CCA CTC CTG GAT CCC CGG ATC-3'; Egr4(F) 5'-TTG AGC TGG GCT TTG AAC A-3'; Egr4(R) $5^{\prime}$-AGA TGC CCG ACA TGA GGT T-3'; Foxd3(F) 5'-CCC ATC ACG GAC AGC CTC AG-3'; Foxd3(R) 5'-TAG GCT GTT CTT GGG CTT GC-3'; Pou3f1(F) 5'-GCG AGC ACT CGG ACG AGG AT-3'; Pou3f1(R) 5'-CGC AGA CGG CTT GGG ACA CT-3'; Dlx2(F)5' GCC TCA CCC AAA CTC AGG T-3'; Dlx2(R)5'-AGG CAC AAG GAG GAG AAG C-3'; Lta(F) $5^{\prime}$-ATG GCA TCC TGA AAC CTG-3'; Lta(R) 5'-CCT GGG AGT AGA CAA AGT AGA
G-3'; Tshz2(F) 5'-TCC AGT CCC AAC TCA AGC-3'; Tshz2(R) 5'-CCA GGT CAG AAA GCA GGT-3'; Disp2(F)5'-CCT TTG AAC GCT TTG ACG-3'; Disp2(R) 5'-GCC AGG TTG CCA TGA GTA-3'; Erbb4(F) 5'-GCC CTC AAC CAG TTT CGT-3'; Erbb4(R) 5'-AGC AGC CTC CAG CAC ATT-3'; Hhip(F) 5'-CCC ATC GGC TCT TCA TTC TA-3'; Hhip(R) $5^{\prime}$-CCT TTC GTC TCC TCC CTT TA- $3^{\prime}$; Ihh(F) $5^{\prime}$-TGA CAG AGA TGG CCA GTG AG-3'; Ihh(R) 5'-AGA GCT CAC CCC CAA CTA CA-3'; Wnt6(F) 5'-GCG GAG ACG ATG TGG ACT TC-3'; Wnt6(R) 5'-ATG CAC GGA TAT CTC CAC GG-3'; Dll1(F) 5' -TCA GAT AAC CCT GAC GGA GGC-3'; Dll1(R) 5'-AGG TAA GAG TTG CCG AGG TCC-3'; Dll3(F) 5'-GAT GCC TTT TAC CTG GGC CTG-3'; Dll3(R) 5'-ATC GAA GCC CGT AGA ATC CC-3'; Dll4(F) $5^{\prime}-$ CAG TTG CCC TTC AAT TTC ACC T-3'; Dll4(R)5'-AGC CTT GGA TGA TGA TTT GGC-3'; Figf(F) 5'-CCC ATC GCT CCA CCA GAT TT-3'; Figf(R) 5'-CGC ATG TCT CTC TAG GGC TG-3'; Dkk1(F) $5^{\prime}$-TAT GAG GGC GGG AAC AAG-3'; Dkk1(R)5'-GAT GAT CGG AGG CAG ACG-3'; Wnt4(F) 5'-AGG AGT GCC AAT ACC AGT TCC-3'; Wnt4(R) $5^{\prime}$-TGT GAG AAG GCT ACG CCA TA- $3^{\prime}$; $\beta$-actin (F) $5^{\prime}$-ACG GCC AGG TCA TCA CTA TTG-3'; $\beta$-actin (R) $5^{\prime}$-AGG GGC CGG ACT CAT CGT A-3'.

\section{Sphere Assay and Differentiation Assay}

The flow-sorted cells were diluted to 2 cells/ $\mu$ l in DMEM/F12 medium with $1 \% \quad \mathrm{~N} 2$ (Invitrogen, 17502-048), 2\% B27 (Invitrogen, 17504-044), EGF (20 ng/ml; Sigma, E9644), IGF (50 ng/ml, Sigma, I8779), heparan sulfate (20 ng/ml, Sigma, H4777), $\beta$-FGF (10 ng/ml, Sigma, F0291), and 0.1\% ampicillin (Sigma, A9518-5G) and cultured in Costar ultra-low attachment dishes (Costar, 3599) for 5 days and then passaged to the next generation.

For the differentiation assay, we differentiated both flowsorted cells and spheres. In the cell-differentiation assay, the flow-sorted Lgr5+ progenitors and Lgr5- SCs were cultured to a density of 50 cells $/ \mu$ l on laminin-coated plates using DMEM/F12 medium with 1\% N2 (Invitrogen, 17502-048), 2\% B27 (Invitrogen, 17504-044), EGF (20 ng/ml; Sigma, E9644), IGF (50 ng/ml, Sigma, I8779), heparan sulfate (20 ng/ml, Sigma, H4777), $\beta$-FGF (10 ng/ml, Sigma, F0291), and 0.1\% ampicillin (Sigma, A9518) for 10 days. EdU (10 $\mu \mathrm{M}$, Invitrogen, C10340) was added during the culture in order to label the dividing cells. In the sphere-differentiation assay, the spheres were plated on laminin-coated four-well dishes and cultured in DMEM/F12 medium with 1\% N2 (Invitrogen, 17502-048), 2\% B27 (Invitrogen, 17504-044), and 0.1\% ampicillin (Sigma, A9518) for 10 days.

\section{RNA Extraction for RNA-Seq Analysis}

Approximately 5,000 Lgr5+ HC progenitors and 5,000 Lgr5SCs were isolated by FACS and split into three fractions for separate replicates. RNA-Seq libraries of FACS-purified cells were generated using the SMART-Seq v4 Ultra Low Input RNA Kit for Sequencing and the Illumina mRNA-Seq Sample Prep Kit. FACSpurified cells were suspended in $10 \times$ lysis buffer. First strand and second strand CDNA synthesis, adaptor ligation, and PCR amplification were performed using the Illumina mRNA-Seq 
Sample Prep Kit. SPRI beads (Ampure XP, Beckman) were used in each purification step after RNA fragmentation for size selection. All libraries were analyzed for quality and concentration using an Agilent Bioanalyzer. Sequencing was performed using the Illumina HiSeq2500 150-bp Paired-End Platform, and FASTQ files of paired-end read files were generated.

\section{Data Analysis}

RNA-Seq reads in the FASTQ files were trimmed using Trimmomatic. Clean reads were mapped to the mouse reference genome (mm9) using TopHat (Trapnell and Schatz, 2009) followed by transcript assembly and differential gene expression analysis using Cufflinks. Genes and transcripts were annotated using the RefGene database (NCBI). Genes with $p$-values $<0.05$ were marked as significant. To assess the extent of functional enrichment, we performed gene ontology (GO) analysis with the functional annotation tool DAVID 6.7 (Huang da et al., 2009), which determines whether biological processes are enriched within a list of genes.

\section{Statistical Analysis}

All of the data are shown as the mean $\pm \mathrm{SD}$, and statistical analyses were conducted using GraphPad Prism6 software. For all experiments, $n$ represents the number of replicates, and at least three individual experiments were conducted. Two-tailed, unpaired Student's $t$-tests were used to determine statistical significance when comparing two groups. A value of $p<0.05$ was considered to be statistically significant.

\section{RESULTS}

\section{Lgr5+ HC Progenitors Generate More HCs Compared to the Lgr5- SCs In Vitro}

Lgr5-EGFP-CreERT2 transgenic mice were used to report Lgr5 expression with EGFP (Hume et al., 2007). Lgr5-EGFP is expressed in a subset of SCs, including third-row Deiters' cells, inner pillar cells, and inner phalangeal cells (Chai et al., 2011). To separate the Lgr5+ HC progenitors from the other Lgr5- SCs, we crossed Lgr5-EGFP-CreERT2/Rosa26-tdTomato mice with Sox2-CreERT2/Rosa26-tdTomato mice to generate Lgr5-EGFPCreERT2/Sox2-CreERT2/Rosa26-tdTomato triple-positive mice among the offspring. Tamoxifen was injected at P2, and the cochleae were harvested at P4 (Figure 1A). Several previous studies have noted that the apical turn of the cochlea has greater $\mathrm{HC}$ regeneration ability than the basal turn (Chai et al., 2012; Cox et al., 2014), so to avoid the gene expression differences between the apical and basal turns (Waqas et al., 2016a) we used the middle turn of the cochlea for all of the experiments and analyses. For flow cytometry, we first used the tdTomato channel to sort out all the tdTomato+ cells (Red), which represent all of the Sox $2+$ SCs. From these tdTomato+ cells, we used the FITC channel to isolate the EGFP+ cells, which were the Lgr5+ progenitors, and the rest of the tdTomato + but EGFP - cells were the Lgr5- SCs (Figure 1A). The Lgr5+ progenitors expressed both tdTomato and EGFP and thus were labeled in yellow in
Figure 1. Using this strategy, we could separate the purified Lgr5+ progenitors from the other Lgr5- SCs (Figure 1B).

To determine the HC regeneration capability of Lgr5+ progenitors and Lgr5- SCs, we cultured 5,000 cells in laminincoated 4-well dishes at a density of 50 cells/ $\mu 1$ for 10 days in serum-free medium. We added $10 \mu \mathrm{M}$ EdU to the culture medium from day 4 to 7 during culture to label the mitotically regenerated HCs (Figure 1C). After 10 days of culture, the cells were immunostained with the HC marker Myo7a. We found that the Lgr5+ progenitors generated significantly more Myo7a+ colonies and total colonies than the Lgr5- SCs $\left(^{* *} p<0.01\right.$, $* * * p<0.001, n=3$ ) (Figures 1D-H and Supplementary Figures S1A,B), while the number of Myo7a- colonies was significantly greater for the Lgr5- SCs $(* * p<0.01, n=3)$ (Figure 1H). Isolated Lgr5+ progenitor SCs generated HCs through both direct differentiation and mitotic regeneration. In the differentiation assay, the HCs inside of the colonies represent the mitotically regenerated HCs, and the HCs outside of the colonies represent the directly differentiated HCs. Next, we characterized and counted the Myo7a+ cells inside and outside of the epithelial colonies and found that Lgr5+ progenitors generated significantly more Myo7a $+\mathrm{HCs}$ both inside and outside of the colony than the Lgr5- SCs $\left({ }^{*} p<0.05,{ }^{* *} p<0.01\right.$, $n=3$ ) (Figure 1I). When we counted the Myo7a+/EdU+ cells, we found that the majority of the Myo7a+/EdU+ cells were inside of the colonies and only a few of the Myo7a+/EdU+ cells were outside of the colonies (Figures 1D-G,J) and that Lgr5+ progenitors generated significantly more Myo7a+/EdU+ HCs both inside and outside of the colonies than the Lgr5- SCs $\left({ }^{*} p<0.05,{ }^{* *} p<0.01,{ }^{* * *} p<0.001, n=3\right)$ (Figure 1J).

To further verify our findings, we used multiple $\mathrm{HC}$ markers, including Myo6 and parvalbumin (PV), to label the newly regenerated HCs. We found that all of the Myo7a+ cells were also Myo6+ and PV+ in both the population of HCs regenerated from Lgr5+ progenitors and the population generated from Lgr5- SCs (Supplementary Figures S1C-F). To further investigate the bundle morphology of newly regenerated HCs, we used the common hair bundle markers phalloidin and espin1. We found that 68.7 and $66.3 \%$ of newly regenerated HCs had hair bundles from Lgr5+ progenitors and Lgr5SCs, respectively (Supplementary Figures S1E,F,G,H). To test whether the newly regenerated HCs have mechanosensory transduction function, we performed additional FM1-43 dye experiments. Almost all of the newly regenerated HCs from both Lgr5+ progenitors and Lgr5- SCs could take up FM1-43 dye, suggesting that the majority of the newly regenerated HCs had the mechanosensory transduction function (Supplementary Figures S1I,J). Taken together, these results suggest that Lgr5+ progenitors generate significantly more HCs than the Lgr5- SCs in vitro.

\section{Lgr5+ Progenitors have Higher Sphere-Forming Ability than Lgr5- SCs}

Sphere-forming assays have been used to evaluate cell proliferation in many studies ( $\mathrm{Li}$ et al., 2003; Sinkkonen et al., 2011; Chai et al., 2012; Shi et al., 2012; Jan et al., 2013; 

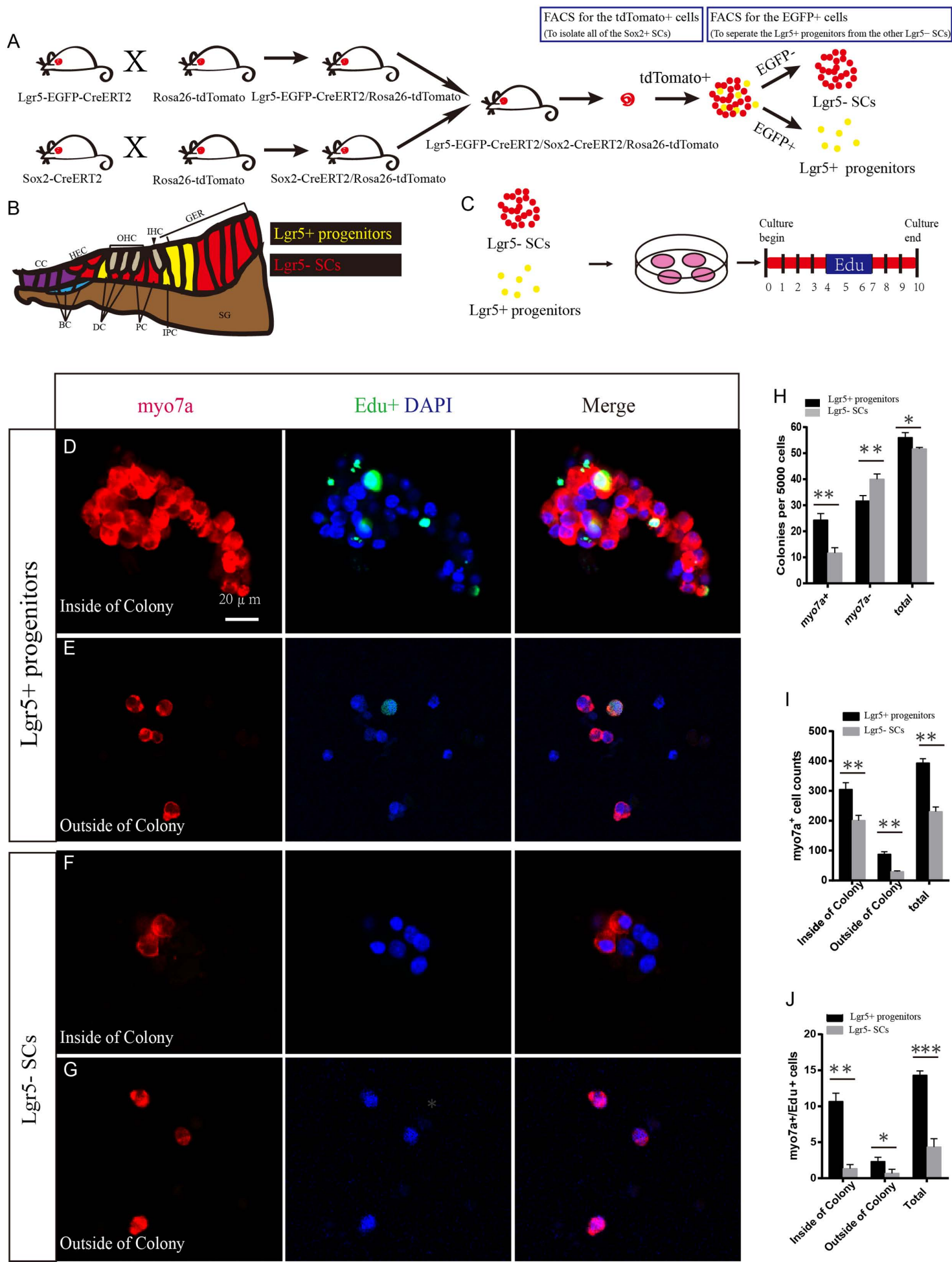

FIGURE 1 | Lgr5+ HC progenitors generate more HCs compared to Lgr5- SCs in vitro. (A) We crossed the Lgr5-EGFP-CreERT2/Rosa26-tdTomato mice with Sox2-CreERT2/Rosa26-tdTomato mice to get the Lgr5-EGFP-CreERT2/Sox2-CreERT2/Rosa26-tdTomato triple-positive mice. We used the tdTomato channel to sort out all of the Sox2+ SCs, and then we used the FITC channel to separate the tdTomato/EGFP double-positive Lgr5+ progenitors from the other tdTomato+ but EGFP-SCs. Because Lgr5+ progenitors expressed both EGFP and tdTomato, the yellow dots represent the Lgr5+ progenitor cells. (B) Schematic depicting cell types in the PO-P3 cochlea. Lgr5+ progenitor cells were labeled in yellow and the Lgr5- SCs were labeled in red. DC, Deiters' cells; PC, inner pillar cells; IPC, inner phalangeal cells; GER, the lateral greater epithelial ridge; BC, Boettcher cells; CC, Claudius cells; HEC, Hensen's cells; SGN, spiral 


\section{FIGURE 1 | Continued}

ganglion neuron. (C) We cultured the sorted EGFP+ cells at 50 cells/ $\mu$ l and added EdU from day 4 to 7 . The total culture time was 10 days. (D) Lgr5+ progenitors generated a large number of Myo7a+ cells on the inside of the colony, and some of them were co-labeled with EdU. (E) Lgr5+ progenitors also generated some Myo7a+ cells outside of the colony. (F,G) Lgr5- SCs generated fewer Myo7a+ cells inside of the colony and outside of the colony, and few of them were co-labeled with EdU. (H) The number of colonies in each well per 5,000 cells. The Lgr5+ progenitors formed around 24 Myo7a+ colonies and 30 Myo7a- colonies, while the Lgr5- SCs formed around 11 Myo7a+ colonies and 40 Myo7a- colonies. (I) Both inside and outside of the colony, Lgr5+ progenitors formed more Myo7a+ cells compared with Lgr5-SCs. (J) In Lgr5+ progenitors, some of the Myo7a+ cells were co-labeled with EdU inside of the colony and outside of the colony $\left({ }^{*} p<0.05\right.$, ${ }^{* *} p<0.01,{ }^{* * *} p<0.001, n=3$. Scale bars are $20 \mu \mathrm{m}$ in $\left.\mathbf{D}-\mathbf{G}\right)$.

Lu et al., 2016). To determine the sphere-forming ability of Lgr5+ progenitors and Lgr5- SCs, we purified the two cell populations by flow cytometry, cultured 200 sorted cells at a density of 2 cells/ $\mu$ in a Costar ultra-low attachment plate for 5 days, and counted the number of spheres before passage to the next generation (Figure 2A). We found that Lgr5+ progenitors formed significantly more spheres than the Lgr5SCs $(p<0.05, n=3)$, but there was no difference in the size of the spheres (Figures 2B,C). To further evaluate the HC regeneration ability of these spheres, we isolated the spheres derived from Lgr5+ progenitors and the Lgr5- SCs from the first generation and differentiated the spheres for 10 days. EdU was added from day 4 to 7 during the culture, and the cells were stained for Myo7a after culture (Figure 2D). We counted the Myo7a+ HCs in each differentiated sphere as well as the total number of Myo7a+ HCs. We found that each sphere that originated from Lgr5+ progenitors generated almost 8 times as many Myo7a+ HCs than spheres from the Lgr5- SCs (Figures 2E-H), and the total spheres originating from Lgr5+ progenitors gave rise to significantly more total Myo7a+ HCs than those originating from the Lgr5- SCs (Figures 2I,J). In a similar manner, we used Myo6 and PV to label the newly regenerated HCs, and we found that all of the Myo7a+ cells were also Myo6+ and PV+ in both groups (Supplementary Figures S2A-D). We also used phalloidin and espin1 to show the bundle morphology of newly regenerated HCs, and we found that 61.4 and $58.3 \%$ of newly regenerated HCs had hair bundles from Lgr5+ progenitors and Lgr5-SCs, respectively (Supplementary Figures S2A',B',G,H,G',H'). We also performed the FM1-43 dye experiments to test whether the newly regenerated HCs had the mechanosensory transduction function. We found that almost all of the newly regenerated HCs from both groups could take up FM1-43 dye, suggesting that the majority of the newly regenerated HCs had the mechanosensory transduction function (Supplementary Figures S2E,F). These results showed that Lgr5+ progenitors had a greater ability to proliferate and to generate HCs than the other Lgr5- SCs.

\section{Analysis of RNA-Seq Results}

We performed RNA-Seq analysis to identify differences in gene expression between Lgr5+ progenitors and Lgr5- SCs. Between 30.8 and 47.7 million paired-end reads were obtained for each sample, with $58.1-75.5 \%$ of the read pairs mapping correctly to the reference genome (mouse $\mathrm{mm} 9$ ). The expression of every gene was measured by FPKM (Fragments Per Kilobase of transcript per Million fragments mapped), and we filtered out genes with low expression (FPKM $<1)$. Three replicates of each population showed high reproducibility (Pearson's $r=0.923-0.957$ for the Lgr5- SC populations and 0.941 for the Lgr5+ progenitor populations) (Figure 3A). After excluding genes with FPKM below the baseline, 13,997 and 12,392 genes were expressed in the Lgr5+ progenitors and the Lgr5- SCs, respectively, and 11,225 of these genes were expressed in both cell populations (Figure 3B).

\section{Genes Enriched in Lgr5+ Progenitors or Lgr5- SCs}

In order to characterize the gene-expression profiles in Lgr5+ progenitors and Lgr5- SCs, we explored the most abundantly expressed genes in both populations. Figure 3C shows the expression levels for the top 200 most abundant genes in Lgr5-SCs. For comparison, expression levels for the same transcripts in the Lgr5+ progenitors and abundance rankings for these transcripts are also illustrated. Figure 3D similarly shows the 200 most abundant transcripts in Lgr5+ progenitors compared to the same transcripts in Lgr5- SCs. As shown in both figures, the majority of the transcripts that are richly expressed in one population are also abundantly expressed in the other. However, among the most abundantly expressed genes, $M b p, P m p 22$, and H1fO were significantly highly expressed in Lgr5- SCs, and Pcp4, Acbd7, 4930170H14Rik, and Gm6537 were significantly highly expressed in Lgr5+ progenitors. None of these genes have been previously reported to be expressed in the inner ear.

\section{Differentially Expressed Genes in Lgr5+ Progenitors and Lgr5- SCs}

To determine which genes are differentially expressed in Lgr5+ progenitors and Lgr5- SCs, we compared the expression levels of all of the transcripts in Lgr5+ progenitors with those of Lgr5- SCs and selected the top differentially expressed genes (Figure 4A). Differentially expressed genes were categorized as those whose expression levels were above background and at least 2 -fold different between the Lgr5+ progenitors and Lgr5- SCs $(p<0.05)$. We found 1,826 genes that were differentially highly expressed in Lgr5- SCs and 986 genes that were differentially highly expressed in Lgr5+ progenitors. Figures 4B,C show the top 150 differentially expressed genes in Lgr5+ progenitors and Lgr5- SCs. The functions of some of the differentially expressed genes have been reported previously. Some of the genes that are highly expressed in Lgr5+ progenitors have been reported to play roles in inner ear HC development, ear morphogenesis, and neuron projection, including Cib2 (Ahmed et al., 2013), Epha4 (Defourny et al., 2013), Espn 

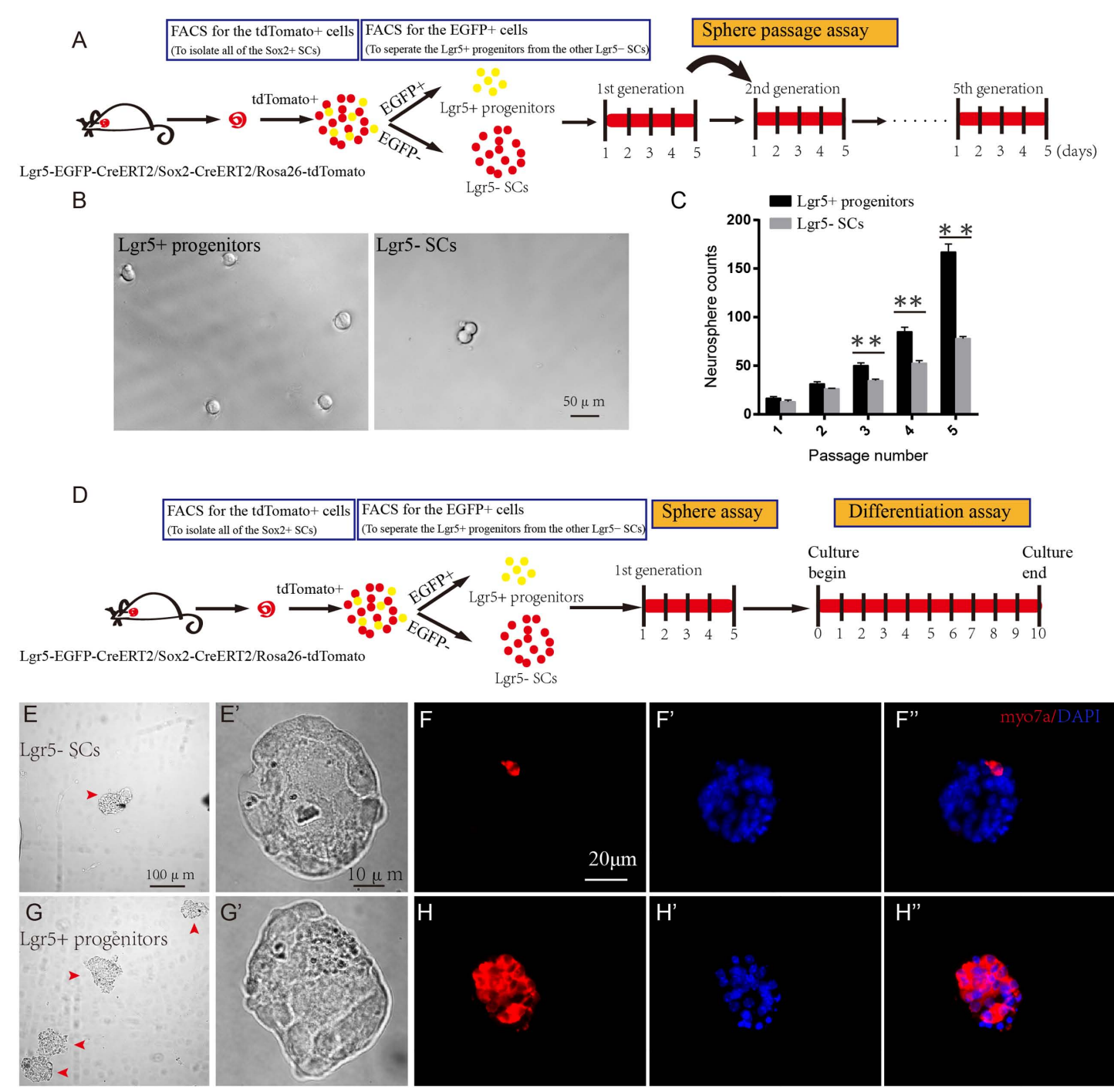

I

$\mathrm{J}$
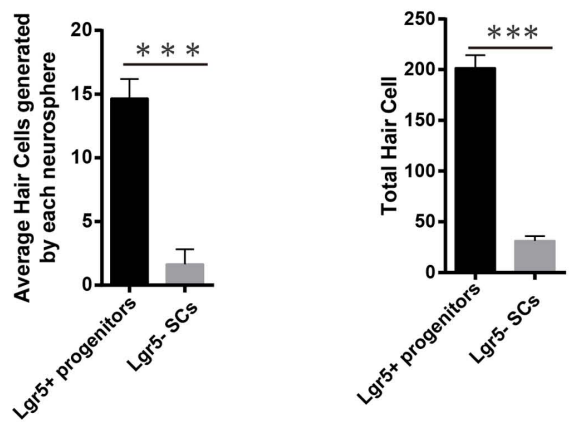

FIGURE 2 | Lgr5+ progenitors have greater sphere-forming ability than Lgr5- SCs. (A) Tamoxifen was injected at P3, and the mice were harvested at P5. FACS was used to isolate the Lgr5+ progenitors and Lgr5- SCs, and these cells were cultured for 5 days and passaged to the next generation. (B) Lgr5+ progenitors generated significantly more spheres than Lgr5- SCs. (C) Lgr5+ progenitors had a significantly higher rate of expansion than Lgr5- SCs. (D) The cultured cells in the first generation were used for the differentiation assay. (E,E') The Differential Interference Contrast microscope configuration (DIC) pictures show the low magnification (E) and high magnification (E') images of the spheres formed by Lgr5- SCs. (G,G') The DIC pictures show the low magnification (G) and high magnification (G') images of the spheres formed by Lgr5+ progenitors. (F,F') An Lgr5- sphere stained with the HC marker Myo7a. (F') Represents the sphere stained with DAPI, (F') represents merged image. (H,H') An Lgr5+ sphere stained with the HC marker Myo7a. (H') Represents the sphere stained with DAPI and (H") shows the merged image. (I) The average number of HCs generated by each sphere. (J) The total number of hair cells generated by $200 \mathrm{Lgr} 5+$ progenitors or Lgr5- SCs. ${ }^{*} p<0.05, * * p<0.01,{ }^{* * *} p<0.001, n=3$. Scale bars are $20 \mu \mathrm{m}$ in $\mathbf{( E , F ) . ~}$ 

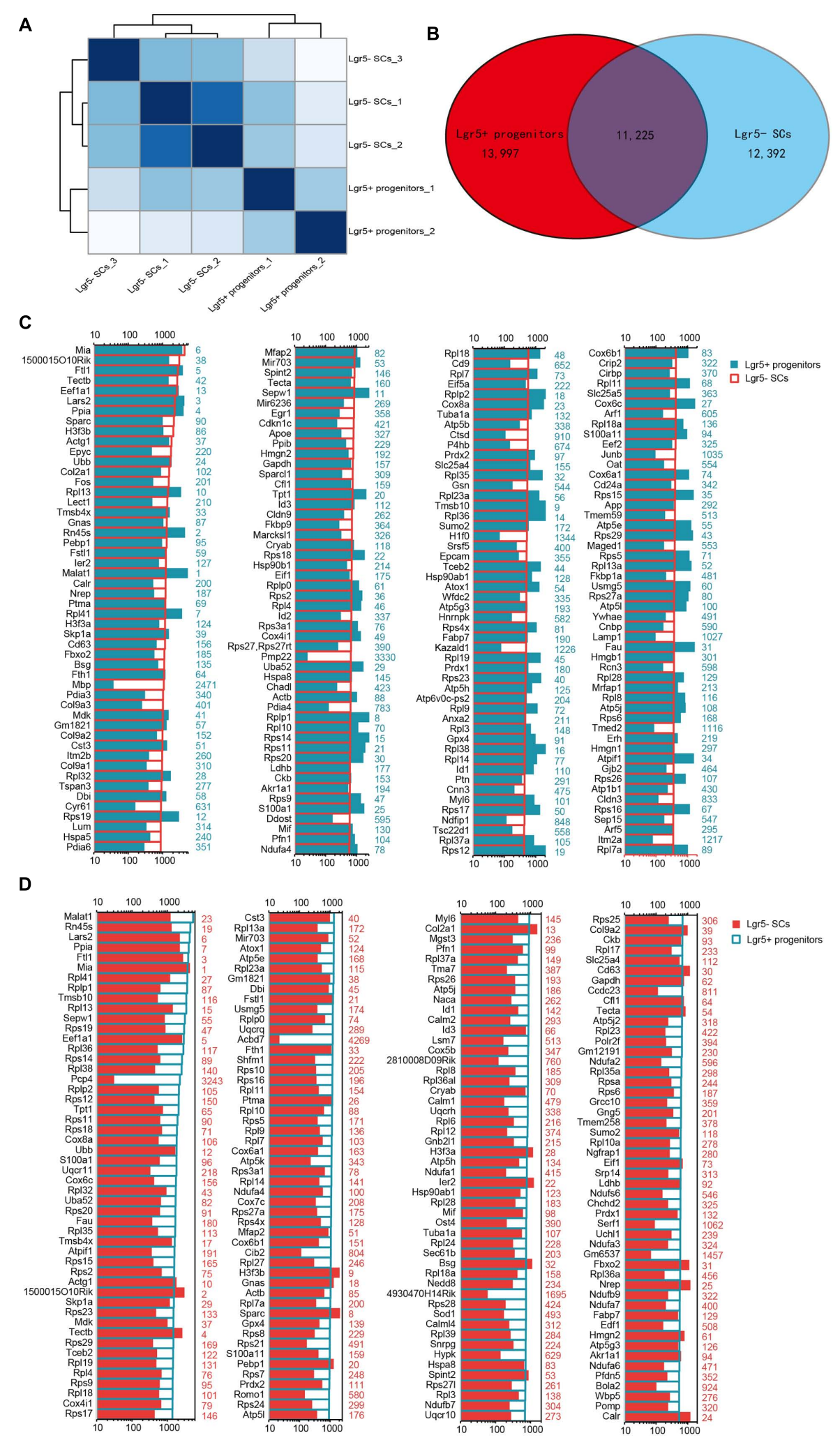

FIGURE 3 | Top 200 genes highly expressed in Lgr5+ progenitors and Lgr5- SCs. (A) Sample clustering analysis for all replicates of Lgr5+ progenitors and Lgr5- SCs. (B) Venn diagram showing genes expressed in Lgr5+ progenitors and Lgr5- SCs. (C) The top 200 genes that are highly expressed in Lgr5- SCs ranked in descending order. The number in blue on the right side of each panel represents the same gene ranking in Lgr5+ progenitors. (D) The top 200 genes highly expressed in Lgr5+ progenitors ranked in descending order. The number in red on the right side of each panel represents the same gene ranking in Lgr5- SCs. 


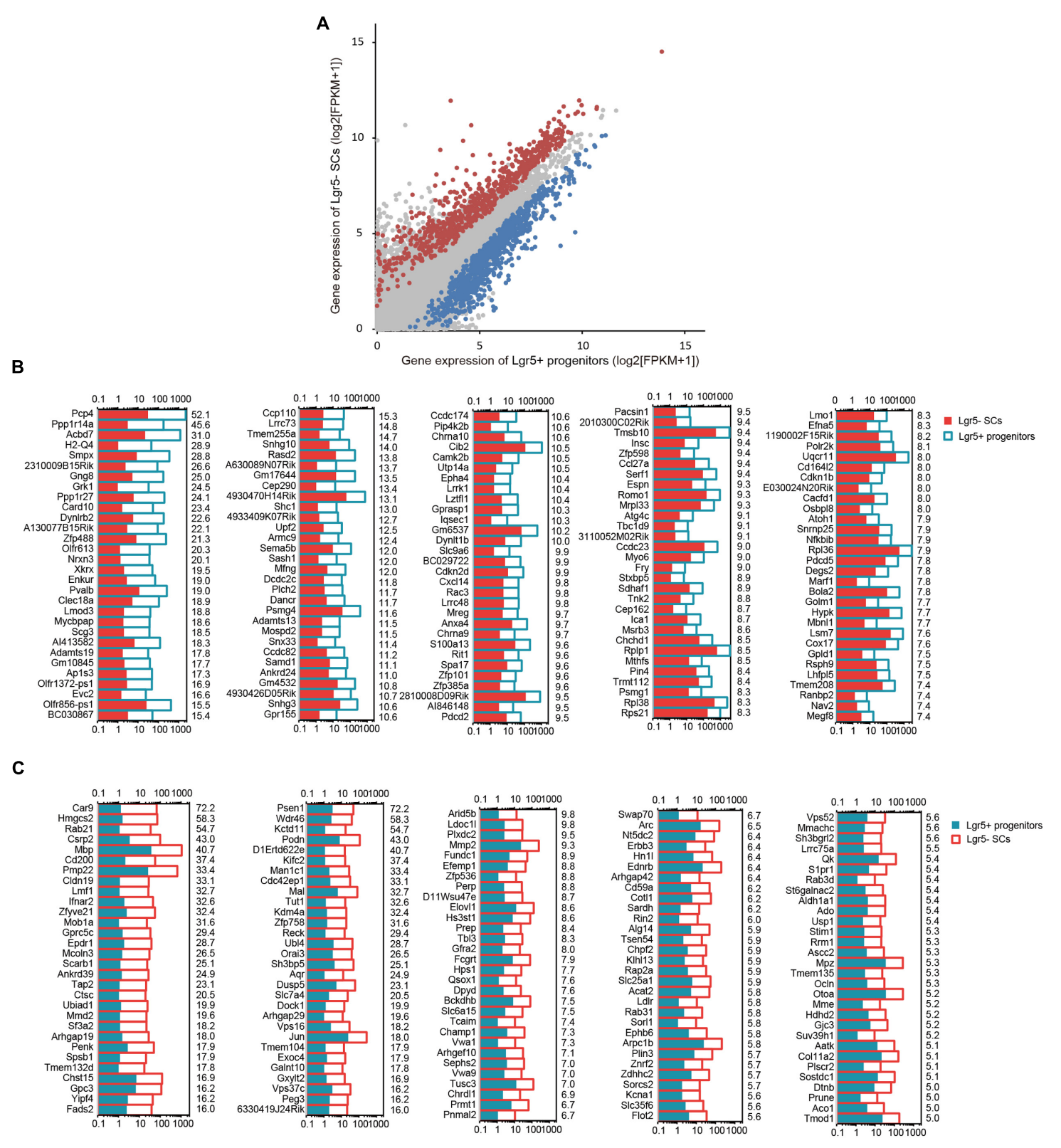

FIGURE 4 | The differentially expressed genes in Lgr5+ progenitors and Lgr5- SCs. (A) All expressed transcripts in Lgr5- SCs and Lgr5+ progenitors. The blue dots represent the highly differentially expressed genes in Lgr5+ progenitors, the red dots represent the highly differentially expressed genes in Lgr5- SCs, and the gray dots represent the genes that are highly expressed in both Lgr5- SCs and Lgr5+ progenitors. (B) The top 150 highly differentially expressed genes in Lgr5+ progenitors ranked in descending order. The number on the right of each panel represents the fold difference in expression for Lgr $5+$ progenitors versus Lgr5- SCs. (C) The top 150 highly differentially expressed genes in Lgr5- SCs ranked in descending order. The number on the right of each panel represents the fold difference in expression for Lgr5- SCs versus Lgr5+ progenitors.

(Sekerkova et al., 2006), Lhfpl5 (Zhao et al., 2014), Smpx (Huebner et al., 2011; Schraders et al., 2011), and Lmo1 (Deng et al., 2006), and this supports our notion that Lgr5+ progenitors have a much greater potential to generate more sensory HCs in the neonatal cochlea. However, a significant number of the differentially expressed genes have not been characterized before and need to be further studied in the future.

\section{Cell Cycle Analysis}

Lgr5+ progenitors had significantly greater proliferation and mitotic $\mathrm{HC}$ regeneration ability than the other Lgr5SCs; however, the detailed mechanism behind this difference remains unknown. To identify the possible genes regulating the cell cycling of Lgr5+ progenitors, we used RNA-Seq analysis to compare the expression of genes regulating the cell cycle and cell proliferation in Lgr5+ progenitors and 

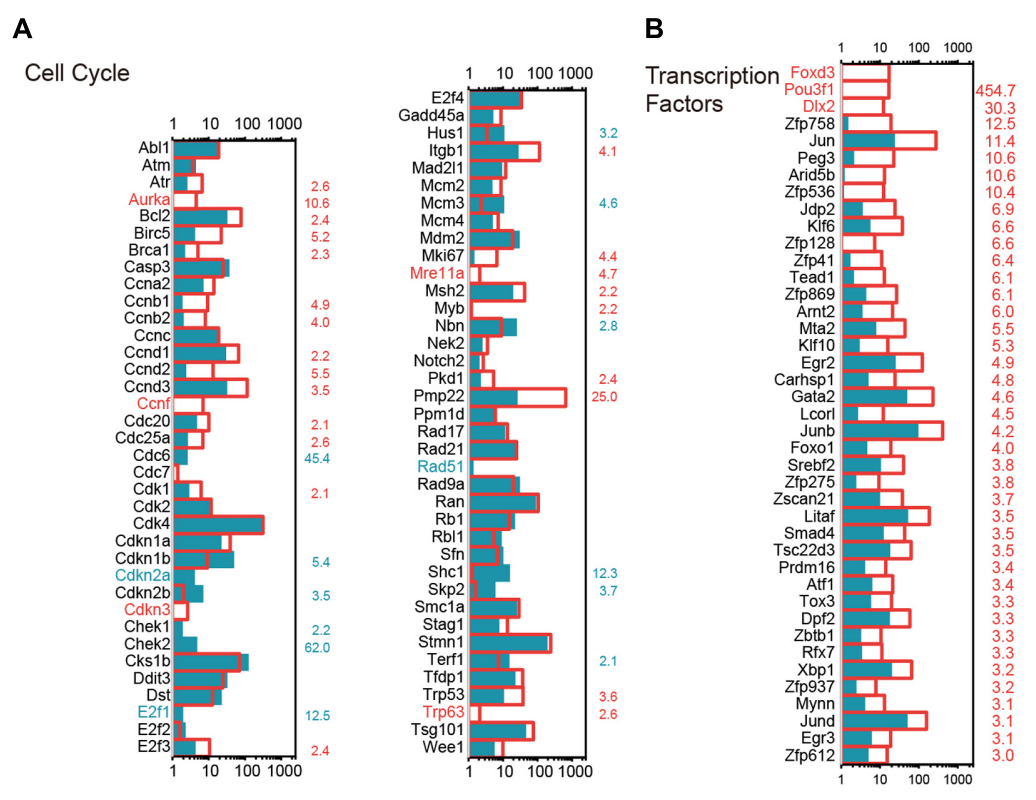

B
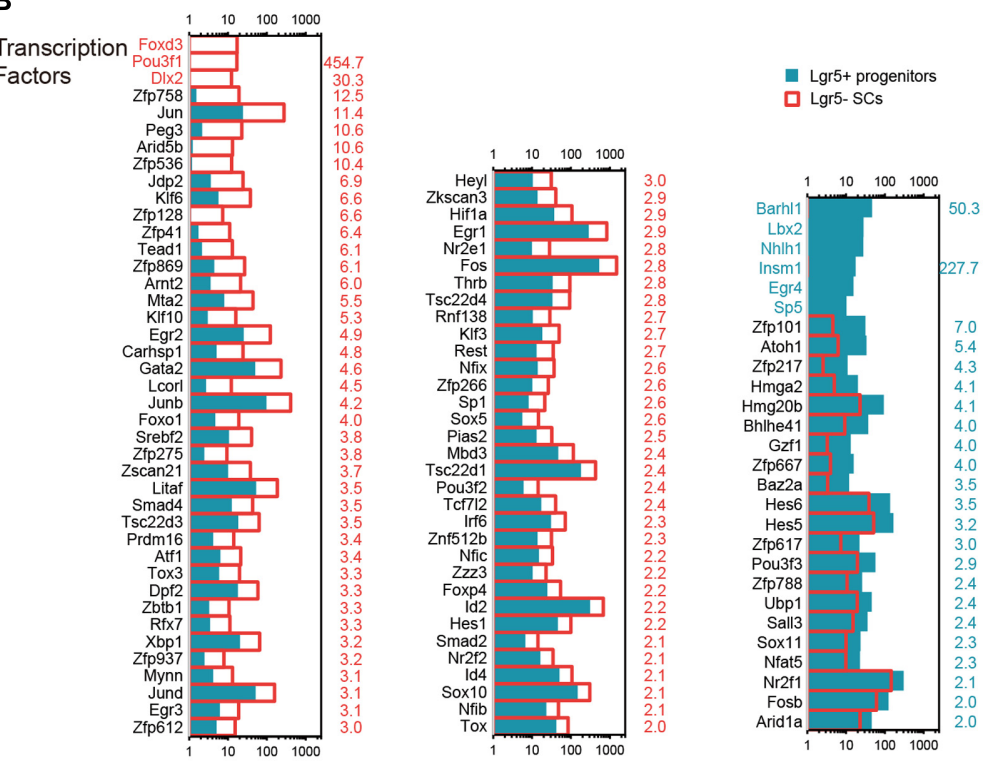

C

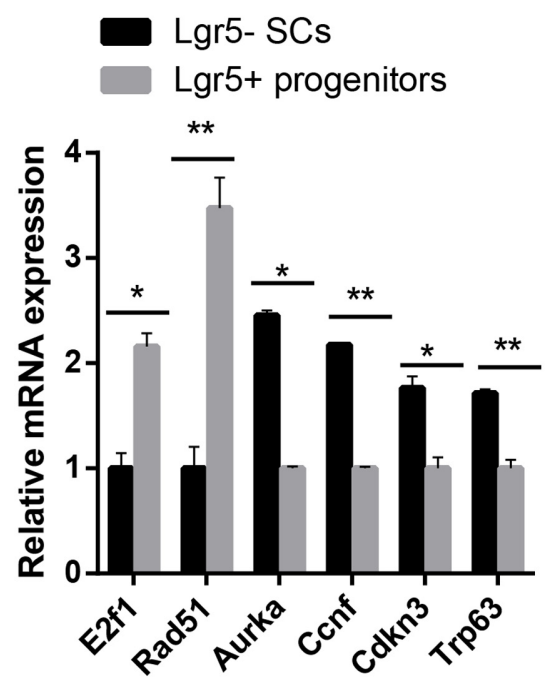

D
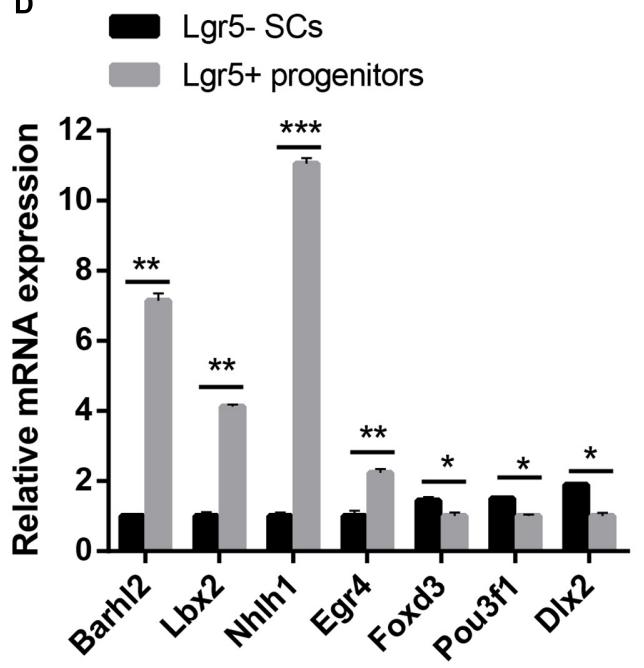

FIGURE 5 | Cell cycle genes and transcription factors in Lgr5- SCs and Lgr5+ progenitors. (A) The expression of 80 genes involved in the cell cycle in Lgr5- SCs and Lgr5+ progenitors. (B) The expression of 83 transcription factor genes in Lgr5 - SCs and Lgr5+ progenitors. In (A,B), the gene names in red on the left of each panel represent the genes that are uniquely detected in Lgr5- SCs, and the blue names represent the genes that are uniquely detected in Lgr5+ progenitors. The number in red on the right of each panel represents the fold difference in expression for Lgr5- SCs versus Lgr5+ progenitors, and the blue number on the right of each panel represents the fold difference in expression for Lgr5+ progenitors versus Lgr5- SCs. (C) q-PCR analysis of the cell cycle genes.

(D) $q$-PCR analysis of the transcription factors. ${ }^{*} p<0.05,{ }^{* *} p<0.01,{ }^{* * *} p<0.001, n=3$.

Lgr5- SCs. It has been reported that over 1,000 cell cycle genes might exist in the mammalian cell (Forrest et al., 2003), and we examined the expression of 80 genes known to be involved in the cell cycle and that are commonly assayed in cell cycle PCR arrays. We found that Cdkn1b, Cdkn2a, E2f1, Rad51, and Shc1 were significantly highly expressed in Lgr5+ progenitors and that Aurka, Bcl2, Ccnd1, Cond2, Cond3, Conf, Cdkn3, Itgb1, Mki67, Mrella, Msh2, $\operatorname{Pmp22}$, and $\operatorname{Trp63}$ were significantly highly expressed in
Lgr5- SCs (Figure 5A). However, most of the differentially expressed cell cycle-regulating genes that we identified in Lgr5+ progenitors and Lgr5- SCs have not been characterized before in the inner ear and need to be further studied. We performed q-PCR to confirm the RNA-Seq data, and the results were consistent with the microarray analysis data (Figure 5C). We did not detect the expression of Aurka or Mrella, possibly because of their low gene expression. 
A

EGF

D

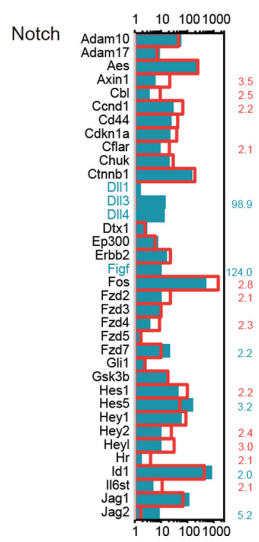

B

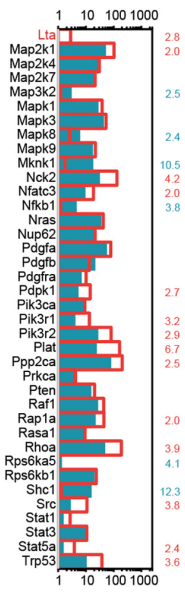

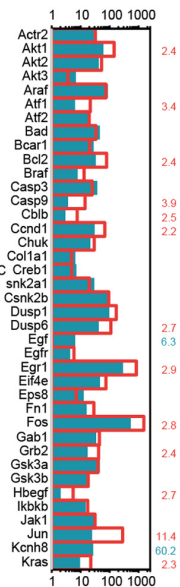

Hippo

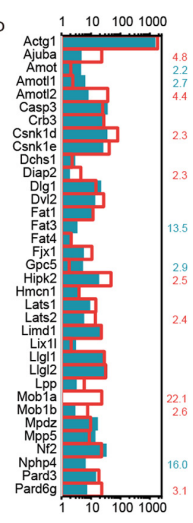

E

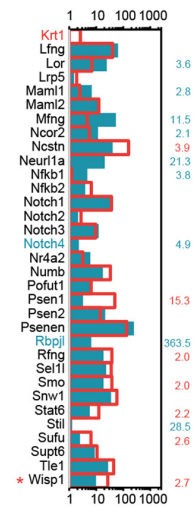

C

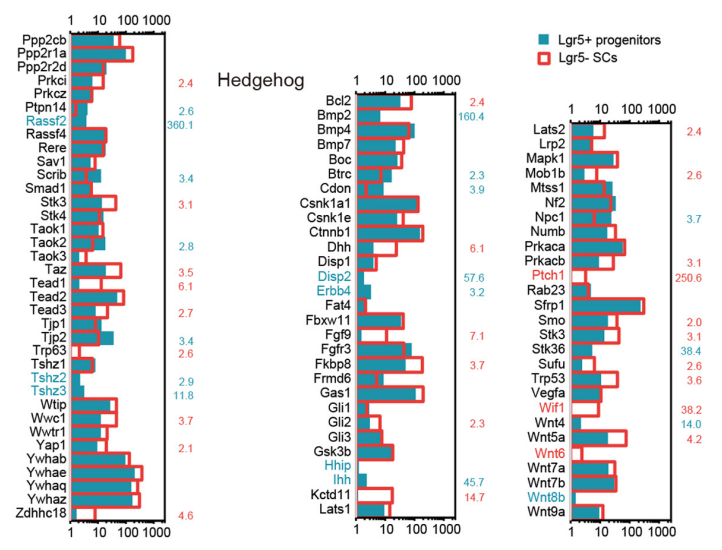

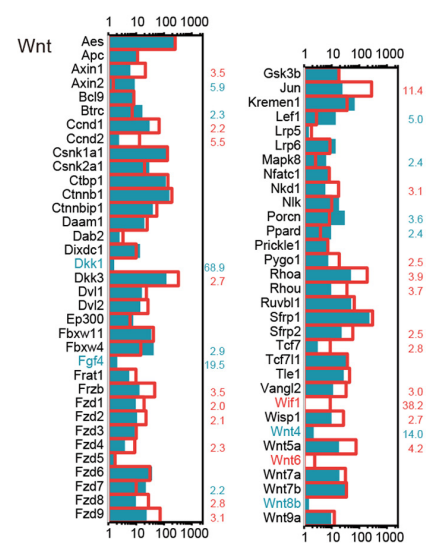

- Lgr5-SCs

F

\section{Lgr5+ progenitors}

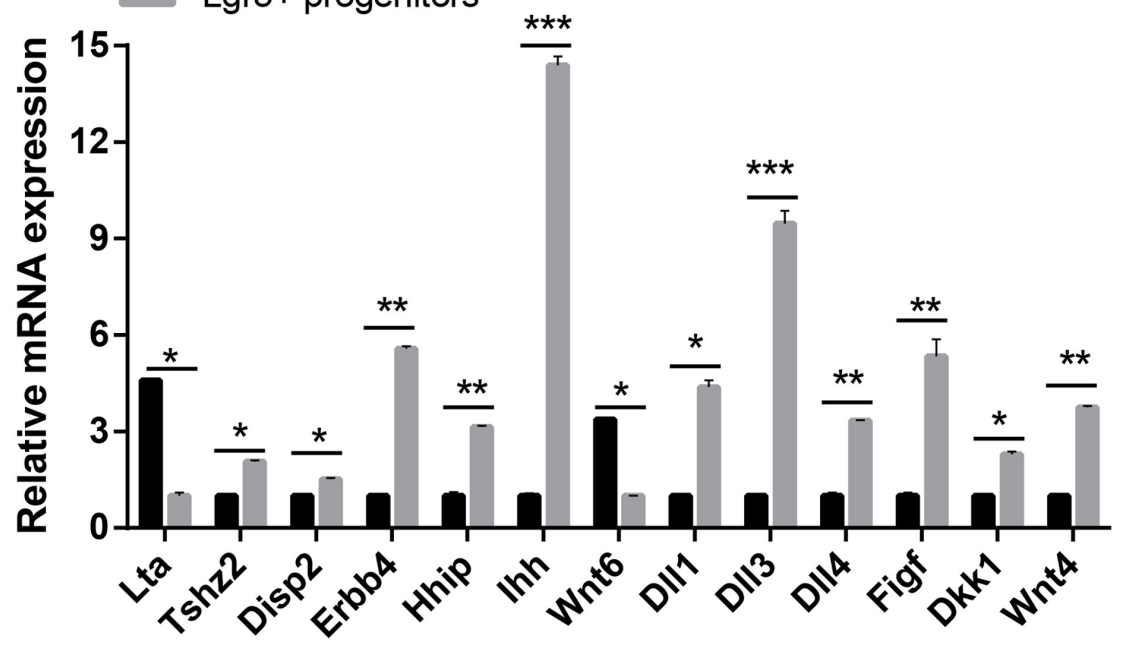

FIGURE 6 | Signaling pathway genes in Lgr5- SCs and Lgr5+ progenitors. The differentially expressed genes in Lgr5- SCs and Lgr5+ progenitors that are involved in (A) EGF, (B) Hippo, (C) Hedgehog, (D) Notch, and (E) Wnt signaling pathways. The gene names in red on the left of each panel represent the genes uniquely detected in Lgr5- SCs, and the names in blue represent the genes uniquely detected in Lgr5+ progenitors. The number in red on the right of each panel represents the fold difference in expression for Lgr5- SCs versus Lgr5+ progenitors, and the number in blue on the right of each panel represents the fold difference in expression for Lgr5+ progenitors versus Lgr5- SCs. (F) q-PCR analysis of the EGF, Hippo, Hedgehog, Notch, and Wnt signaling pathway genes. ${ }^{*} p<0.05$, ${ }^{* *} p<0.01,{ }^{* * *} p<0.001, n=3$. 


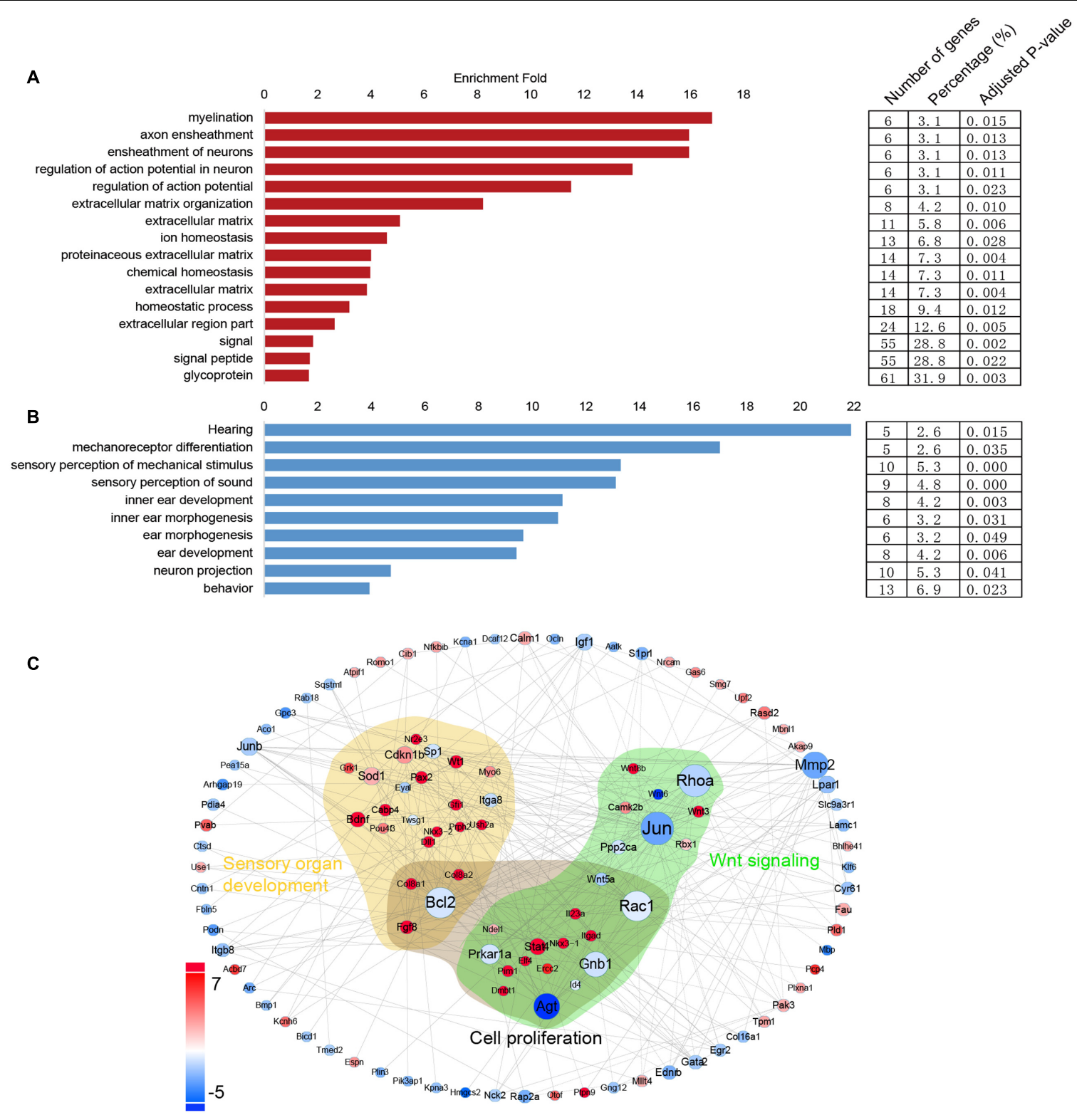

FIGURE 7| Gene ontology and network analysis of the genes differentially expressed in Lgr5+ progenitors and Lgr5- SCs and the PCA analysis. (A) The functions of genes upregulated in Lgr5- SCs. (B) The function of genes upregulated in Lgr5+ progenitors. (C) The STRING protein-protein interaction analysis of genes that are differentially expressed in Lgr5+ progenitors (blue) and Lgr5- SCs (red).

\section{Transcription Factors Analysis}

Transcription factors (TFs) are proteins that bind to either enhancer or promoter regions of genes thereby controlling the expression level of these target genes. TFs are involved in various processes, including inner ear development and $\mathrm{HC}$ regeneration. To determine which TFs might be involved in regulating HC regeneration, we examined the expression of 1,324 TFs in the mouse genome in Lgr5+ progenitors and Lgr5- SCs. Figure 5B shows the 83 significantly differentially expressed TFs in Lgr5+ progenitors and Lgr5- SCs $(p<0.05$, fold change $>2)$. We found that the genes for six TFs (Barhl1, Lbx2, Nhlh1, Insm 1,
Egr4, and Sp5) were richly expressed in Lgr5+ progenitors but not detected in the Lgr5- SCs at all. Some of the TF genes that were highly expressed in Lgr5+ progenitors have been reported to play roles in promoting $\mathrm{HC}$ fate and patterning regulation during inner ear development, including Atoh1, Barhl1, Hmga2, Pou3f3, and Sox11 (Mutai et al., 2009; Chonko et al., 2013; Smeti et al., 2014; Gnedevaa and Hudspeth, 2015), and some of the TF genes that are highly expressed in Lgr5- SCs have been reported to play critical roles in regulating cell survival and apoptosis in the inner ear, including Gata2 (Haugas et al., 2010), Hifla (Chung et al., 2011), Thrb (Ng et al., 2001), Jun 
(Sanz et al., 1999), Smad4 (Yang et al., 2009), and Hes1 (Kelley, 2006). We performed q-PCR to confirm the RNA-Seq data, and the results were consistent with the microarray analysis data (Figure 5D). We did not detect the expression of $S p 5$, possibly as a result of the low gene expression. We have identified many TFs that have not been characterized before, and their involvement in regulating the $\mathrm{HC}$ regeneration capacity of Lgr5+ progenitors and Lgr5- SCs should be investigated in the future.

\section{Signaling Pathway Analysis}

Several major signaling pathways play important roles in regulating cell proliferation and $\mathrm{HC}$ regeneration, including the EGF, Hedgehog, Wnt, Notch, and Hippo pathways. To determine which signaling pathway factors are involved in regulating the proliferation and $\mathrm{HC}$ regeneration ability of Lgr5+ progenitors and Lgr5- SCs, we measured over 1000 genes, many of which had significant differences in expression (Figure 6). The pathway genes that are highly expressed in Lgr5+ progenitors include Kcnh8, Mknk1, Shc1, Cdon, Disp2, Erbb4, Hhip, Ihh, Npc1, Dll1, Dll3, Dll4, Figf, Hes5, Mfng, Neurl1a, Notch4, Rbpjl, Dkk1, Fgf4, Wnt4, Wnt8b, Fat3, Rassf2, Tjp2, Tshz2, and Tshz3. Among them, Axin2 (Jan et al., 2013; Jansson et al., 2015), Egf (Lou et al., 2015), Wnt4 (Alvarado et al., 2011), Tjp2 (Kim et al., 2014), Dll1 (Kiernan et al., 2005; Chrysostomou et al., 2012), and Dll3 (Hartman et al., 2007) have already been reported in the inner ear. Some of the pathway genes that are highly expressed in Lgr5-SCs include Akt1, Atf1, Bcl2, Ccnd1, Dusp6, Egr1, Fos, Grb2, Jun, Kras, Lta, Pik3r1, Pik3r2, Plat, Ppp2ca, Rhoa, Dhh, Fgf9, Fkbp8, Kctd11, Prkacb, Ptch1, Stk3, Fos, Hesl, Heyl, Krt1, Ncstn, Psen1, Ccnd2, Dkk3, Fzd8, Fzd9, Fkbp8, Jun, Nkd1, Rhou, Wif1, Frzb, Wnt5a, Wnt6, Ajuba, Amotl2, Csnk1d, Hipk2, Mob1a, Pard6g, Stk3, Wisp1, Taz, Tead1, and Wwc1. Among these, Dusp6 (Urness et al., 2008), Rhoa (Sai et al., 2014), Fgf9 (Huh et al., 2015), Frzb (Qian et al., 2007), Fzd1, Fzd4, Fzd9 (Shah et al., 2009), Fkbp8 (Zak et al., 2011), Hey2 (Benito-Gonzalez and Doetzlhofer, 2014), Src (Andreeva et al., 2014), Smo (Tateya et al., 2013), Vangl2 (Copley et al., 2013), Wnt5a (Qian et al., 2007), Wnt6 (Lillevali et al., 2006), and Wif1 (Dabdoub et al., 2003) have already been reported in the inner ear. Most of the cell-signaling pathway genes have not been characterized before in the inner ear.

We performed q-PCR to confirm the RNA-Seq data, and the result was consistent with the microarray analysis data (Figure 6F). We did not detect the expression of Wnt8, Ptch1, Wif1, Krt1, or Fgf4, possibly as a result of their low gene expression. The different expression of these genes might be involved in regulating the different proliferation and regeneration ability of Lgr5+ progenitors compared to the Lgr5- SCs.

\section{Gene Ontology Analysis of the Differentially Expressed Genes in Lgr5+ Progenitors and Lgr5- SCs}

In order to obtain a comprehensive view of the gene network involved in inner ear HC regeneration, we performed a STRING protein-protein interaction analysis (Franceschini et al., 2013), which assembles the predicted networks of the differentially expressed genes (fold change $>2.0, p<0.01$ ) with the functional categories highlighted by GO analysis (DAVID) (Figure 7C). This integrated GO analysis suggests a complex network of genes that are involved in inner ear HC development and are predicted to participate in regulating inner ear development, cell proliferation, and Wnt signaling. GO analysis was also applied to the genes that are upregulated in Lgr5+ progenitors and Lgr5- SCs (fold change $>2.0, p<0.01$ ) (Figures 7A,B). As shown in Figure 7A, genes upregulated in Lgr5+ progenitors were highly enriched in functional categories such as hearing, mechanoreceptor differentiation, and inner ear development, while the genes upregulated in Lgr5- SCs were slightly enriched in functional categories such as signaling and the extracellular matrix.

\section{DISCUSSION}

In the mouse inner ear, SCs can divide and transdifferentiate into HCs (White et al., 2006; Cox et al., 2014; Lu et al., 2016). Lgr5 is only expressed in a subset of SCs, and it is enriched in the population of HC progenitors (Chai et al., 2011; Shi et al., 2012). The Lgr5+ progenitors have a greater capacity to regenerate HCs both in vitro and in vivo than Lgr5- SCs, and Lgr5+ progenitors can be regulated by Wnt and Notch signaling to regenerate HCs via both direct differentiation and mitotic regeneration (Wang et al., 2015; Li et al., 2016; Lu et al., 2016; Ni et al., 2016; Waqas et al., 2016b). When isolated by FACS, the Lgr5+ progenitors can be passaged for at least five generations (Chai et al., 2012; Shi et al., 2012, 2013; Bramhall et al., 2014; Li et al., 2016; Waqas et al., 2016a; Wu et al., 2016). In this study, we isolated the Lgr5+ progenitors and the other Lgr5- SCs from transgenic mice by flow cytometry. The Lgr5+ progenitors differentiated to form more Myo7a + HCs, and they formed more spheres than Lgr5- SCs. To understand the mechanism behind the different proliferation and $\mathrm{HC}$ regeneration ability of Lgr5+ progenitors compared to the other Lgr5- SCs, we determined the genomewide transcriptional profiles of these two cell populations via RNA-Seq profiling.

\section{Differentially Expressed Genes in Lgr5+ Progenitors and Lgr5- SCs}

Among the top 150 differentially expressed genes, most of them have not been reported in the inner ear, and only a few of them have been described before. The genes that are highly expressed in Lgr5+ progenitors include Cib2, Epha4, Espn, Atoh1, Lhfpl5, Smpx, and Lmo1. Epha4 is expressed in outer HCs and spiral ganglion neurons (SGNs), and it mediates afferent signaling to HCs (Defourny et al., 2013). Lhfpl5 mutation affects tip-link assembly (Zhao et al., 2014), and Smpx is strongly expressed in the sensory epithelium and plays a role in HC formation (Huebner et al., 2011; Schraders et al., 2011). Lmo1 is suggested to play an important role in $\mathrm{HC}$ differentiation and is specifically expressed in cochlear HCs and vestibular HCs during the development of the inner ear (Deng et al., 2006). The genes Cib2 and Espn are involved in the formation of stereocilia in the inner ear, and their disruption can lead to hearing impairment (Sekerkova et al., 2006; 
Ahmed et al., 2013). Our analysis showed that some of the genes that are highly expressed in Lgr5+ progenitors are crucial for $\mathrm{HC}$ formation during inner ear development, and expression of these genes might be the source of the high HC regeneration capabilities of Lgr5+ progenitors.

The set of reported genes that are highly expressed in Lgr5- SCs includes Ednrb, S1pr1, and Tekt2. Ednrb mutation causes syndromic hearing loss due to congenital defects in the melanocytes in the stria vascularis of the inner ear (Matsushima et al., 2014). S1pr1 is expressed in both the organ of Corti and the SGN, and it plays a role in maintaining the function of vestibular and cochlear HCs (Nakayama et al., 2014). Tekt2 is also expressed in HCs and participates in the transient appearance of the microtubule-based kinocilium in the cochlear HCs (Yoon et al., 2011). The genes that are highly expressed in Lgr5- SCs are mainly involved in the function of the cochlea.

Furthermore, we analyzed the cell cycle genes, TF genes, and signaling pathway factor genes that might regulate proliferation and $\mathrm{HC}$ regeneration ability, and we found 8 cell cycle genes, 9 TF genes, and 24 signaling pathway factor genes that are uniquely expressed in either Lgr5+ progenitors or Lgr5- SCs.

\section{Cell Cycle Analysis}

The highly expressed genes in Lgr5+ progenitors include E2f1, Cdkn1b, and Rad51. E2f1 is expressed in the SGN, and mitochondrial reactive oxygen species-mediated E2f1 activation induces apoptosis in the SGN (Raimundo et al., 2012). The $C d k n 1 b$ and Rad51 genes in the auditory sensory epithelium promote the proliferation and formation of supernumerary HCs in the post-natal and adult cochlea (Chen and Segil, 1999; Lowenheim et al., 1999; Oesterle et al., 2014; Walters et al., 2014).

The genes that are highly expressed in Lgr5- SCs include Bcl2, Birc5, Pkd1, Trp63, and Itgb1. Bcl2 knockout mice have high-frequency hearing loss due to a developmental defect in the stapes (Carpinelli et al., 2012; Liu et al., 2014). Birc5 is broadly expressed in the cochlea, and it can protect HCs against damage (Habtemichael et al., 2010). Pkd1 is localized to the $\mathrm{HC}$ stereocilia, and it plays an essential role in stereocilia structure and maintenance (Steigelman et al., 2011). Trp63 is important for normal development of the cochlea by activating the Notch signaling pathway (Terrinoni et al., 2013). Itgb1 is expressed throughout the otic area - including the sensory epithelium and the periodic mesenchyme - during inner ear development (Matilainen et al., 2007). We also found other cell cycle-promoting genes (including Ran, Stmn1, and Smc1a) and cell cycle-inhibiting genes (including E2f4, Rbl1, and $M d m 2$ ) that are abundantly expressed in both cell populations. In addition to these, the other newly identified cell cycle regulatory genes in Lgr5+ progenitors and Lgr5- SCs need to be further characterized.

\section{Transcription Factor Analysis}

The highly expressed TF genes in Lgr5+ progenitors include Barhl1, Atoh1, Hmga2, Pou3f3, and Sox11. Atoh1 promotes cochlear HC survival and differentiation, and Barhl1 is a downstream gene of Atoh1 that is essential for HC maintenance (Chonko et al., 2013). Hmga2 is broadly expressed during inner ear development, which suggests its potential dual role in early differentiation and in the maintenance of both $\mathrm{HC}$ and SC phenotypes (Smeti et al., 2014). Pou3f3 is specifically expressed in SCs and mesenchymal cells, and it is important for the maintenance and functional development of the post-natal cochlea (Mutai et al., 2009). Sox11 promotes the differentiation of SCs into HCs (Gnedevaa and Hudspeth, 2015).

The TF genes that were highly expressed in Lgr5- SCs include Gata2, Hif1a, Thrb, Jun, Smad4, and Hes1. Gata2 is required for vestibular morphogenesis (Haugas et al., 2010). Hif1a and Thrb are expressed in HCs, and high expression of Hifla prevents noise-induced hearing loss (Chung et al., 2011), while a lack of Thrb leads to the developmentally delayed establishment of potassium currents ( $\mathrm{Ng}$ et al., 2001). The Jun gene plays a critical role during inner ear development by mediating apoptosis through the JNK pathway (Sanz et al., 1999). Smad4 is required for inner ear development (Yang et al., 2009), and Hes1 inhibits SC differentiation by decreasing the expression of Atoh1 (Kelley, 2006). Our results suggest that the higher expression of these negative transcriptional regulators might be involved in the reduced proliferation capacity of Lgr5- SCs compared to Lgr5+ progenitors

\section{Signaling Pathway Analysis}

The signaling factor genes that are highly expressed in Lgr5+ progenitors include Axin2, Wnt4, Tjp2, Dll1, and Dll3. Axin2 acts as a Wnt target gene, and its expression in tympanic border cells allows them to behave as HC progenitors (Jan et al., 2013; Jansson et al., 2015). Combined with other growth factors, Egf can protect HCs from ototoxic damage (Lou et al., 2015). Wnt4 is detected in the inner and outer spiral sulcus cells, as well as in the Claudius and Hensen's cells, and downregulation of Wnt4 expression significantly reduces the proliferation of SCs (Alvarado et al., 2011). The Tjp2 gene is mainly expressed in the membrane between the HCs and SCs, and mutation of the Tjp2 gene causes hearing loss (Op de Beeck et al., 2011; Kim et al., 2014). Both Dll1 and Dll3 can repress HC formation and can promote HC differentiation (Kiernan et al., 2005; Hartman et al., 2007; Chrysostomou et al., 2012; Petrovic et al., 2014).

The signaling factor genes that are highly expressed in Lgr5-SCs include Dusp6, Egf, Rhoa, Fgf9, Frzb, Fzd1, Fzd4, Fzd9, Fkbp8, Hey2, Src, Smo, Vangl2, Wnt5a, Wnt6, and Wif1. Dusp6 is expressed in the otic region during embryonic development and acts as a negative feedback regulator of FGF signaling (Urness et al., 2008). Rhoa, Wnt5a, Src, Vangl2, and Wif1 mediate planar cell polarity in the inner ear (Dabdoub et al., 2003; Qian et al., 2007; Copley et al., 2013; Andreeva et al., 2014; Sai et al., 2014), and Src inhibitors protect HCs from noise-induced damage (Bielefeld, 2015). Fgf9 participates in regulating the number of cochlear progenitors and the length of the cochlea through mesenchymal FGFR signaling (Pirvola et al., 2004; Huh et al., 2015). Smo is an effector of Hedgehog signaling that inhibits prosensory cell differentiation into HCs or SCs and delays differentiation in the apical region (Tateya et al., 2013). $F z d 1, F z d 4$, and $F z d 9$ are expressed in adult mouse SGNs, and Fzd9 guides neurite regeneration in SGNs (Shah et al., 2009). Frzb is expressed in the lateral region of the developing organ of Corti 
and regulates stereociliary orientation and cochlear extension (Qian et al., 2007). Fkbp8 is localized in the SGN and is important for the onset of hearing processes in rodents (Zak et al., 2011). Hey 2 is highly expressed in HCs and SCs, and it is critical for maintaining prosensory cells in an undifferentiated state (BenitoGonzalez and Doetzlhofer, 2014). Wnt6 is the first Wnt gene expressed in the otic epithelium at embryonic day 8.5 , and its expression is confined to the dorsal portion of the otic placode (Lillevali et al., 2006). Our analysis shows that the genes that are highly expressed in Lgr5- SCs are mainly involved in the function of the cochlea but not in cell proliferation or HC regeneration.

\section{STRING Prediction of Inner Ear HC Development}

In this protein-protein interaction network, most of the genes in the GO categories of sensory organ development were highly expressed in Lgr5+ progenitors, such as Gfil, Pou4f3, and Pax2, although several genes such as Eya1 and Bcl2 were richly expressed in Lgr5- SCs. It would be interesting to further investigate the involvement of these genes in regulating the progenitor cells.

All of the data we provided in this paper were from the neonatal mouse cochlea. The situation might be completely different in the adult mouse cochlea or in the damaged mouse cochlea, and the approaches to promote $\mathrm{HC}$ regeneration in the neonatal cochlea might not have the same effects in the adult cochlea. Thus, further investigations in the adult cochlea or damaged cochlea need to be performed in the future.

\section{CONCLUSION}

We found that Lgr5+ progenitors have significantly greater proliferation and $\mathrm{HC}$ regeneration ability than Lgr5- SCs. We investigated the transcriptome differences between Lgr5+ progenitors and Lgr5- SCs and found significantly differentially expressed genes that might regulate the Lgr5+ progenitors' proliferation and $\mathrm{HC}$ regeneration capacity. The most interesting of these are the genes that are uniquely expressed in Lgr5+ progenitors but not in Lgr5- SCs. To further analyze the role of differentially expressed genes in $\mathrm{HC}$ regeneration and proliferation, we constructed a STRING prediction map. The transcriptomes of Lgr5+ progenitors and Lgr5- SCs reported here establish a framework for future characterization of the genes that regulate the proliferation and $\mathrm{HC}$ regeneration ability of Lgr5+ progenitors, and these genes might represent new therapeutic targets for $\mathrm{HC}$ regeneration.

\section{AUTHOR CONTRIBUTIONS}

CC, LG, LL, HS, HL, and RC designed the study. CC, LG, HS, $\mathrm{XX}$, and SZ performed the laboratory experiments. RC, CC, LG,
HS, CX, HL, MW, YC, FC, XZ, XG, and MT contributed to critical discussion and data analysis. CC, MW, HS, RC, HL, JG, and LG wrote the paper. All authors read and approved the final manuscript.

\section{ACKNOWLEDGMENTS}

This work was supported by grants from the Major State Basic Research Development Program of China (973 Program; 2015CB965000), the National Natural Science Foundation of China (Nos. 81622013, 81470692, 81230019, 81500790, 81570921, 31500852, 31501194), the Jiangsu Province Natural Science Foundation (BK20150022, BK20150598, BK20140620, BK20160125), the Boehringer Ingelheim Pharma $\mathrm{GmbH}$, the Yingdong Huo Education Foundation, the Fundamental Research Funds for the Central Universities, the Excellent Academic Leader Training Program of the Shanghai Municipal Health System (XBR2013085), the Project of Invigorating Health Care through Science Technology and Education, and the Open Research Funds of the State Key Laboratory of Genetic Engineering Fudan University (SKLGE-1407).

\section{SUPPLEMENTARY MATERIAL}

The Supplementary Material for this article can be found online at: http://journal.frontiersin.org/article/10.3389/fnmol. 2017.00122/full\#supplementary-material

FIGURE S1 | Lgr5+ HC progenitors generate more HCs compared to Lgr5- SCs in vitro. (A,B) After 10 days of culture, the low magnification images of the inside colonies and outside colonies showed that Lgr5+ progenitors generated more Myo7a+ colonies than Lgr5- SCs. (C,D) The HC marker Myo6 labeled the newly regenerated HCs in Lgr5- SCs and Lgr5+ progenitors. All the Myo7a+ cells are also Myo6+ in both the population of HCs regenerated from Lgr5+ cells and the population regenerated from Lgr5- SCs. (E,F) The HC marker PV also labeled the newly regenerated HCs in Lgr5- SCs and Lgr5+ progenitors. All of the Myo7a+ cells are also PV+ in both the population of HCs regenerated from Lgr5+ progenitors and the population regenerated from Lgr5- SCs. (E',F') The hair bundle marker phalloidin in Lgr5+ progenitors and Lgr5- SCs. (G,H) The hair bundle marker espin1 in Lgr5+ progenitors and Lgr5SCs. The yellow arrows show the long bundles and the white arrows show the short bundles. (I,J) The FM1-43 dye staining in Lgr5- SCs and Lgr5+ progenitors. Scale bars are $100 \mu \mathrm{m}$ in (A,B); $2 \mu \mathrm{m}$ in (C-F); and $5 \mu \mathrm{m}$ in (G-J).

FIGURE S2 | Lgr5+ progenitors have greater sphere-forming ability than Lgr5- SCs. (A,B) The HC marker Myo6 labeled the newly regenerated HCs from the spheres formed by Lgr5- SCs and Lgr5+ progenitors. All of the Myo7a+ cells are also Myo6+ in both the population of HCs regenerated from Lgr5+ cells and the population regenerated from Lgr5- SCs. (C,D) The HC marker PV labeled the newly regenerated HCs from the spheres formed by Lgr5- SCs and Lgr5+ progenitors. All of the Myo7a+ cells are also PV+ in both the population of HCs regenerated from Lgr5+ progenitors and the population regenerated from Lgr5SCs. (A',B') The hair bundle marker phalloidin in the spheres formed by Lgr5+ C progenitors and Lgr5- SCs. (E,F) The hair bundle marker espin1 in the spheres formed by Lgr5+ progenitors and Lgr5- SCs. The yellow arrows show the long bundles and the white arrows show the short bundles. (G,H) The FM1-43 dye staining in Lgr5- SCs and Lgr5+ progenitors. Scale bars are $20 \mu \mathrm{m}$ in (A,B); $5 \mu \mathrm{m}$ in (C-F); and $10 \mu \mathrm{m}$ in $\mathbf{( G , H ) . ~}$ 


\section{REFERENCES}

Ahmed, Z. M., Frolenkov, G. I., and Riazuddin, S. (2013). Usher proteins in inner ear structure and function. Physiol. Genomics 45, 987-989. doi: 10.1152/ physiolgenomics.00135.2013

Alvarado, D. M., Hawkins, R. D., Bashiardes, S., Veile, R. A., Ku, Y. C., Powder, K. E., et al. (2011). An RNA interference-based screen of transcription factor genes identifies pathways necessary for sensory regeneration in the avian inner ear. J. Neurosci. 31, 4535-4543. doi: 10.1523/JNEUROSCI.5456-10.2011

Andreeva, A., Lee, J., Lohia, M., Wu, X., Macara, I. G., and Lu, X. (2014). PTK7-Src signaling at epithelial cell contacts mediates spatial organization of actomyosin and planar cell polarity. Dev. Cell 29, 20-33. doi: 10.1016/j.devcel.2014.02.008

Balak, K. J., Corwin, J. T., and Jones, J. E. (1990). Regenerated hair cells can originate from supporting cell progeny: evidence from phototoxicity and laser ablation experiments in the lateral line system. J. Neurosci. 10, 2502-2512.

Barker, N., van Es, J. H., Kuipers, J., Kujala, P., van den Born, M., Cozijnsen, M., et al. (2007). Identification of stem cells in small intestine and colon by marker gene Lgr5. Nature 449, 1003-1007. doi: 10.1038/nature06196

Benito-Gonzalez, A., and Doetzlhofer, A. (2014). Heyl and Hey2 control the spatial and temporal pattern of mammalian auditory hair cell differentiation downstream of Hedgehog signaling. J. Neurosci. 34, 12865-12876. doi: 10.1523/ JNEUROSCI.1494-14.2014

Bermingham-McDonogh, O., and Reh, T. A. (2011). Regulated reprogramming in the regeneration of sensory receptor cells. Neuron 71, 389-405. doi: 10.1016/j. neuron.2011.07.015

Bielefeld, E. C. (2015). Protection from noise-induced hearing loss with Src inhibitors. Drug Discov. Today 20, 760-765. doi: 10.1016/j.drudis.2015.01.010

Bramhall, N. F., Shi, F., Arnold, K., Hochedlinger, K., and Edge, A. S. (2014). Lgr5positive supporting cells generate new hair cells in the postnatal cochlea. Stem Cell Rep. 2, 311-322. doi: 10.1016/j.stemcr.2014.01.008

Carpinelli, M. R., Wise, A. K., Arhatari, B. D., Bouillet, P., Manji, S. S. M., Manning, M. G., et al. (2012). Anti-apoptotic gene $\mathrm{Bcl} 2$ is required for stapes development and hearing. Cell Death Dis. 3:e362 doi: 10.1038/cddis.2012.100.

Chai, R., Kuo, B., Wang, T., Liaw, E. J., Xia, A., Jan, T. A., et al. (2012). Wnt signaling induces proliferation of sensory precursors in the postnatal mouse cochlea. Proc. Natl. Acad. Sci. U.S.A. 109, 8167-8172. doi: 10.1073/pnas. 1202774109

Chai, R., Xia, A., Wang, T., Jan, T. A., Hayashi, T., Bermingham-McDonogh, O., et al. (2011). Dynamic expression of Lgr5, a Wnt target gene, in the developing and mature mouse cochlea. J. Assoc. Res. Otolaryngol. 12, 455-469. doi: 10.1007/ s10162-011-0267-2

Chen, P., and Segil, N. (1999). p27(Kip1) links cell proliferation to morphogenesis in the developing organ of Corti. Development 126, 1581-1590.

Chonko, K. T., Jahan, I., Stone, J., Wright, M. C., Fujiyama, T., Hoshino, M., et al. (2013). Atoh1 directs hair cell differentiation and survival in the late embryonic mouse inner ear. Dev. Biol. 381, 401-410. doi: 10.1016/j.ydbio.2013.06.022

Chrysostomou, E., Gale, J. E., and Daudet, N. (2012). Delta-like 1 and lateral inhibition during hair cell formation in the chicken inner ear: evidence against cis-inhibition. Development 139, 3764-3774. doi: 10.1242/dev.074476

Chung, J. W., Shin, J. E., Han, K. W., Ahn, J. H., Kim, Y. J., Park, J. W., et al. (2011). Up-regulation of hypoxia-inducible factor-1 alpha by cobalt chloride prevents hearing loss in noise-exposed mice. Environ. Toxicol. Pharmacol. 31, 153-159. doi: $10.1016 /$ j.etap.2010.10.002

Copley, C. O., Duncan, J. S., Liu, C., Cheng, H., and Deans, M. R. (2013). Postnatal refinement of auditory hair cell planar polarity deficits occurs in the absence of Vangl2. J. Neurosci. 33, 14001-14016. doi: 10.1523/JNEUROSCI.1307-13.2013

Corwin, J. T., and Cotanche, D. A. (1988). Regeneration of sensory hair cells after acoustic trauma. Science 240, 1772-1774.

Cox, B. C., Chai, R., Lenoir, A., Liu, Z., Zhang, L., Nguyen, D. H., et al. (2014). Spontaneous hair cell regeneration in the neonatal mouse cochlea in vivo. Development 141, 816-829. doi: 10.1242/dev.103036

Dabdoub, A., Donohue, M. J., Brennan, A., Wolf, V., Montcouquiol, M., Sassoon, D. A., et al. (2003). Wnt signaling mediates reorientation of outer hair cell stereociliary bundles in the mammalian cochlea. Development 130, 2375-2384.

Defourny, J., Poirrier, A. L., Lallemend, F., Mateo Sanchez, S., Neef, J., Vanderhaeghen, P., et al. (2013). Ephrin-A5/EphA4 signalling controls specific afferent targeting to cochlear hair cells. Nat. Commun. 4, 1438. doi: 10.1038/ ncomms 2445
Deng, M., Pan, L., Xie, X., and Gan, L. (2006). Differential expression of LIM domain-only (LMO) genes in the developing mouse inner ear. Gene Expr. Patterns 6, 857-863. doi: 10.1016/j.modgep.2006.02.005

Forrest, A. R., Taylor, D., and Grimmond, S. (2003). Exploration of the cellcycle genes found within the RIKEN FANTOM2 data set. Genome Res. 13, 1366-1375. doi: 10.1101/gr.1012403

Franceschini, A., Szklarczyk, D., Frankild, S., Kuhn, M., Simonovic, M., Roth, A., et al. (2013). STRING v9.1: protein-protein interaction networks, with increased coverage and integration. Nucleic Acids Res. 41, D808-D815. doi: 10.1093/nar/gks1094.

Gnedevaa, K., and Hudspeth A. J. (2015). SoxC transcription factors are essential for the development of the inner ear. Proc. Natl. Acad. Sci. U.S.A. 112, 14066 14071. doi: 10.1073/pnas.1517371112

Habtemichael, N., Heinrich, U. R., Knauer, S. K., Schmidtmann, I., Bier, C., Docter, D., et al. (2010). Expression analysis suggests a potential cytoprotective role of Birc5 in the inner ear. Mol. Cell. Neurosci. 45, 297-305. doi: 10.1016/j. mcn.2010.07.003

Hartman, B. H., Hayashi, T., Nelson, B. R., Bermingham-McDonogh, O., and Reh, T. A. (2007). Dll3 is expressed in developing hair cells in the mammalian cochlea. Dev. Dyn. 236, 2875-2883. doi: 10.1002/dvdy.21307

Haugas, M., Lillevali, K., Hakanen, J., and Salminen, M. (2010). Gata2 is required for the development of inner ear semicircular ducts and the surrounding perilymphatic space. Dev. Dyn. 239, 2452-2469. doi: 10.1002/dvdy. 22373

Hegarty, S. V., Sullivan, A. M., and O’Keeffe, G. W. (2015). Zeb2: a multifunctional regulator of nervous system development. Prog. Neurobiol. 132, 81-95. doi: 10.1016/j.pneurobio.2015.07.001

Huang da, W., Sherman, B. T., and Lempicki, R. A. (2009). Systematic and integrative analysis of large gene lists using DAVID bioinformatics resources. Nat. Protoc. 4, 44-57. doi: 10.1038/nprot.2008.211

Huebner, A. K., Gandia, M., Frommolt, P., Maak, A., Wicklein, E. M., Thiele, H., et al. (2011). Nonsense mutations in SMPX, encoding a protein responsive to physical force, result in X-chromosomal hearing loss. Am. J. Hum. Genet. 88, 621-627. doi: 10.1016/j.ajhg.2011.04.007

Huh, S. H., Warchol, M. E., and Ornitz, D. M. (2015). Cochlear progenitor number is controlled through mesenchymal FGF receptor signaling. Elife 4:e05921. doi: 10.7554/eLife.05921

Hume, C. R., Bratt, D. L., and Oesterle, E. C. (2007). Expression of LHX3 and SOX2 during mouse inner ear development. Gene Expr. Patterns 7, 798-807. doi: 10.1016/j.modgep.2007.05.002

Jaks, V., Barker, N., Kasper, M., van Es, J. H., Snippert, H. J., Clevers, H., et al. (2008). Lgr5 marks cycling, yet long-lived, hair follicle stem cells. Nat. Genet. 40, 1291-1299. doi: 10.1038/ng.239

Jan, T. A., Chai, R., Sayyid, Z. N., van Amerongen, R., Xia, A., Wang, T., et al. (2013). Tympanic border cells are Wnt-responsive and can act as progenitors for postnatal mouse cochlear cells. Development 140, 1196-1206. doi: 10.1242/ dev.087528

Jansson, L., Kim, G. S., and Cheng, A. G. (2015). Making sense of Wnt signalinglinking hair cell regeneration to development. Front. Cell Neurosci. 9:66. doi: $10.3389 /$ fncel.2015.00066

Kelley, M. W. (2006). Regulation of cell fate in the sensory epithelia of the inner ear. Nat. Rev. Neurosci. 7, 837-849. doi: 10.1038/nrn1987

Kiernan, A. E., Cordes, R., Kopan, R., Gossler, A., and Gridley, T. (2005). The Notch ligands DLL1 and JAG2 act synergistically to regulate hair cell development in the mammalian inner ear. Development 132, 4353-4362. doi: 10.1242/dev. 02002

Kim, M. A., Kim, Y. R., Sagong, B., Cho, H. J., Bae, J. W., Kim, J., et al. (2014). Genetic analysis of genes related to tight junction function in the Korean population with non-syndromic hearing loss. PLoS ONE 9:e95646. doi: 10.1371/ journal.pone.0095646

Li, H., Liu, H., and Heller, S. (2003). Pluripotent stem cells from the adult mouse inner ear. Nat. Med. 9, 1293-1299. doi: 10.1038/nm925

Li, W., You, D., Chen, Y., Chai, R., and Li, H. (2016). Regeneration of hair cells in the mammalian vestibular system. Front. Med. 10, 143-151. doi: 10.1007/ s11684-016-0451-1

Lillevali, K., Haugas, M., Matilainen, T., Pussinen, C., Karis, A., and Salminen, M. (2006). Gata3 is required for early morphogenesis and Fgf10 expression during otic development. Mech. Dev. 123, 415-429. doi: 10.1016/j.mod.2006.04.007 
Liu, H., Pecka, J. L., Zhang, Q., Soukup, G. A., Beisel, K. W., and He, D. Z. (2014). Characterization of transcriptomes of cochlear inner and outer hair cells. J. Neurosci. 34, 11085-11095. doi: 10.1523/jneurosci.1690-14.2014

Lou, X., Yuan, H., Xie, J., Wang, X., Yang, L., and Zhang, Y. (2015). Growth factors have a protective effect on neomycin-induced hair cell loss. Cell Biol. Int. 39, 65-73. doi: 10.1002/cbin.10347

Lowenheim, H., Furness, D. N., Kil, J., Zinn, C., Gultig, K., Fero, M. L., et al. (1999). Gene disruption of p27(Kip1) allows cell proliferation in the postnatal and adult organ of corti. Proc. Natl. Acad. Sci. U.S.A. 96, 4084-4088.

Lu, X., Sun, S., Qi, J., Li, W., Liu, L., Zhang, Y., et al. (2016). Bmil regulates the proliferation of cochlear supporting cells via the canonical Wnt signaling pathway. Mol. Neurobiol. 54, 1326-1339. doi: 10.1007/s12035-016-9686-8

Madisen, L., Zwingman, T. A., Sunkin, S. M., Oh, S. W., Zariwala, H. A., Gu, H., et al. (2010). A robust and high-throughput Cre reporting and characterization system for the whole mouse brain. Nat. Neurosci. 13, 133-140. doi: 10.1038/nn. 2467

Matilainen, T., Haugas, M., Kreidberg, J. A., and Salminen, M. (2007). Analysis of Netrin 1 receptors during inner ear development. Int. J. Dev. Biol. 51, 409-413. doi: $10.1387 /$ ijdb.072273tm

Matsushima, Y., Shinkai, Y., Kobayashi, Y., Sakamoto, M., Kunieda, T., and Tachibana, M. (2014). A mouse model of Waardenburg syndrome type 4 with a new spontaneous mutation of the endothelin-B receptor gene. Mamm. Genome 13, 30-35. doi: 10.1007/s00335-001-3038-2

Mutai, H., Nagashima, R., Sugitani, Y., Noda, T., Fujii, M., and Matsunaga, T. (2009). Expression of Pou3f3/Brn-1 and its genomic methylation in developing auditory epithelium. Dev. Neurobiol. 69, 913-930. doi: 10.1002/dneu.20746

Nakayama, M., Tabuchi, K., Hoshino, T., Nakamagoe, M., Nishimura, B., and Hara, A. (2014). The influence of sphingosine-1-phosphate receptor antagonists on gentamicin-induced hair cell loss of the rat cochlea. Neurosci. Lett. 561, 91-95. doi: 10.1016/j.neulet.2013.12.063

Ng, L., Rüsch, A. A. L., Nordström, K. E. L., and Vennström, B. F. D. (2001). Suppression of the deafness and thyroid dysfunction in Thrb-null mice by an independent mutation in the Thra thyroid hormone receptor $\alpha$ gene. Hum. Mol. Genet. 10, 2701-2708.

Ni, W., Zeng, S., Li, W., Chen, Y., Zhang, S., Tang, M., et al. (2016). Wnt activation followed by Notch inhibition promotes mitotic hair cell regeneration in the postnatal mouse cochlea. Oncotarget 7, 66754-66768. doi: 10.18632/oncotarget. 11479

Oesterle, E. C., Campbell, S., Taylor, R. R., Forge, A., and Hume, C. R. (2008). Sox2 and JAGGED1 expression in normal and drug-damaged adult mouse inner ear. J. Assoc. Res. Otolaryngol. 9, 65-89. doi: 10.1007/s10162-007-0106-7

Oesterle, E. C., Chien, W.-M., Campbell, S., Nellimarla, P., and Fero, M. L. (2014). p27Kiplis required to maintain proliferative quiescence in the adult cochlea and pituitary. Cell Cycle 10, 1237-1248. doi: 10.4161/cc.10.8.15301

Op de Beeck, K., Schacht, J., and Van Camp, G. (2011). Apoptosis in acquired and genetic hearing impairment: the programmed death of the hair cell. Hear. Res. 281, 18-27. doi: 10.1016/j.heares.2011.07.002

Petrovic, J., Formosa-Jordan, P., Luna-Escalante, J. C., Abello, G., Ibanes, M., Neves, J., et al. (2014). Ligand-dependent Notch signaling strength orchestrates lateral induction and lateral inhibition in the developing inner ear. Development 141, 2313-2324. doi: 10.1242/dev.108100

Pirvola, U., Zhang, X., Mantela, J., Ornitz, D. M., and Ylikoski, J. (2004). Fgf9 signaling regulates inner ear morphogenesis through epithelial-mesenchymal interactions. Dev. Biol. 273, 350-360. doi: 10.1016/j.ydbio.2004.06.010

Qian, D., Jones, C., Rzadzinska, A., Mark, S., Zhang, X. H., Steel, K. P., et al. (2007). Wnt5a functions in planar cell polarity regulation in mice. Dev. Biol. 306, 121-133. doi: 10.1016/j.ydbio.2007.03.011

Raimundo, N., Song, L., Shutt, T. E., McKay, S. E., Cotney, J., Guan, M. X., et al. (2012). Mitochondrial stress engages E2F1 apoptotic signaling to cause deafness. Cell 148, 716-726. doi: 10.1016/j.cell.2011.12.027

Roberson, D. F., Weisleder, P., Bohrer, P. S., and Rubel, E. W. (1992). Ongoing production of sensory cells in the vestibular epithelium of the chick. Hear. Res. $57,166-174$.

Sai, X. R., Yonemura, S., and Ladher, R. K. (2014). Junctionally restricted RhoA activity is necessary for apical constriction during phase 2 inner ear placode invagination. Dev. Biol. 394, 206-216. doi: 10.1016/j.ydbio.2014.08.022

Sanz, C., Leon, Y., Canon, S., Alvarez, L., Giraldez, F., and Varela-Nieto, I. (1999). Pattern of expression of the jun family of transcription factors during the early development of the inner ear: implications in apoptosis. J. Cell Sci. 112 (Pt 22), 3967-3974.

Schraders, M., Haas, S. A., Weegerink, N. J. D., Oostrik, J., Hu, H., Hoefsloot, L. H., et al. (2011). Next-generation sequencing identifies mutations of SMPX, which encodes the small muscle protein, $\mathrm{X}$-linked, as a cause of progressive hearing impairment. Am. J. Hum. Genet. 88, 628-634. doi: 10.1016/j.ajhg.2011.04.012

Sekerkova, G., Zheng, L., Loomis, P. A., Mugnaini, E., and Bartles, J. R. (2006). Espins and the actin cytoskeleton of hair cell stereocilia and sensory cell microvilli. Cell. Mol. Life Sci. 63, 2329-2341. doi: 10.1007/s00018-006-6148-x

Shah, S. M., Kang, Y. J., Christensen, B. L., Feng, A. S., and Kollmar, R. (2009). Expression of Wnt receptors in adult spiral ganglion neurons: frizzled 9 localization at growth cones of regenerating neurites. Neuroscience 164, 478-487. doi: 10.1016/j.neuroscience.2009.08.049

Shi, F., Kempfle, J. S., and Edge, A. S. (2012). Wnt-responsive lgr5-expressing stem cells are hair cell progenitors in the cochlea. J. Neurosci. 32, 9639-9648. doi: 10.1523/JNEUROSCI.1064-12.2012

Shi, F. X., Hua, L. X., and Edge, A. S. B. (2013). Generation of hair cells in neonatal mice by beta-catenin overexpression in Lgr5-positive cochlear progenitors. Proc. Natl. Acad. Sci. U.S.A. 110, 13851-13856. doi: 10.1073/pnas.121 9952110

Sinkkonen, S. T., Chai, R., Jan, T. A., Hartman, B. H., Laske, R. D., Gahlen, F., et al. (2011). Intrinsic regenerative potential of murine cochlear supporting cells. Sci. Rep. 1:26. doi: 10.1038/srep00026

Smeti, I., Watabe, I., Savary, E., Fontbonne, A., and Zine, A. (2014). HMGA2, the architectural transcription factor high mobility group, is expressed in the developing and mature mouse cochlea. PLOS ONE 9:e88757. doi: 10.1371/ journal.pone.0088757

Steigelman, K. A., Lelli, A., Wu, X., Gao, J., Lin, S., Piontek, K., et al. (2011). Polycystin-1 is required for stereocilia structure but not for mechanotransduction in inner ear hair cells. J. Neurosci. 31, 12241-12250. doi: 10.1523/JNEUROSCI.6531-10.2011.

Stone, J. S., and Cotanche, D. A. (2007). Hair cell regeneration in the avian auditory epithelium. Int. J. Dev. Biol. 51, 633-647. doi: 10.1387/ijdb.072408js

Tateya, T., Imayoshi, I., Tateya, I., Hamaguchi, K., Torii, H., Ito, J., et al. (2013). Hedgehog signaling regulates prosensory cell properties during the basal-toapical wave of hair cell differentiation in the mammalian cochlea. Development 140, 3848-3857. doi: 10.1242/dev.095398

Terrinoni, A., Serra, V., Bruno, E., Strasser, A., Valente, E., Flores, E. R., et al. (2013). Role of p63 and the Notch pathway in cochlea development and sensorineural deafness. Proc. Natl. Acad. Sci. U.S.A. 110, 7300-7305. doi: 10. 1073/pnas. 1214498110

Trapnell, C., and Schatz, M. C. (2009). Optimizing data intensive GPGPU computations for DNA sequence alignment. Parallel Comput. 35, 429-440. doi: 10.1016/j.parco.2009.05.002.

Urness, L. D., Li, C., Wang, X., and Mansour, S. L. (2008). Expression of ERK signaling inhibitors Dusp6, Dusp7, and Dusp9 during mouse ear development. Dev. Dyn. 237, 163-169. doi: 10.1002/dvdy.21380

Walters, B. J., Liu, Z., Crabtree, M., Coak, E., Cox, B. C., and Zuo, J. (2014). Auditory hair cell-specific deletion of p27Kip1 in postnatal mice promotes cellautonomous generation of new hair cells and normal hearing. J. Neurosci. 34, 15751-15763. doi: 10.1523/JNEUROSCI.3200-14.2014

Wang, T., Chai, R., Kim, G. S., Pham, N., Jansson, L., Nguyen, D. H., et al. (2015). Lgr5+ cells regenerate hair cells via proliferation and direct transdifferentiation in damaged neonatal mouse utricle. Nat. Commun. 6, 6613. doi: 10.1038/ ncomms7613

Waqas, M., Guo, L., Zhang, S., Chen, Y., Zhang, X., Wang, L., et al. (2016a). Characterization of Lgr5+ progenitor cell transcriptomes in the apical and basal turns of the mouse cochlea. Oncotarget 7, 41123-41141. doi: 10.18632/ oncotarget.8636

Waqas, M., Zhang, S., He, Z., Tang, M., and Chai, R. (2016b). Role of Wnt and Notch signaling in regulating hair cell regeneration in the cochlea. Front. Med. 10, 237-249. doi: 10.1007/s11684-016-0464-9

Warchol, M. E. (2011). Sensory regeneration in the vertebrate inner ear: differences at the levels of cells and species. Hear. Res. 273, 72-79. doi: 10.1016/j.heares. 2010.05.004

White, P. M., Doetzlhofer, A., Lee, Y. S., Groves, A. K., and Segil, N. (2006). Mammalian cochlear supporting cells can divide and trans-differentiate into hair cells. Nature 441, 984-987. doi: 10.1038/nature04849 
Wu, J., Li, W., Lin, C., Chen, Y., Cheng, C., Sun, S., et al. (2016). Co-regulation of the Notch and Wnt signaling pathways promotes supporting cell proliferation and hair cell regeneration in mouse utricles. Sci. Rep. 6:29418. doi: 10.1038/ srep29418.

Yang, S. M., Hou, Z. H., Yang, G., Zhang, J. S., Hu, Y. Y., Sun, J. H., et al. (2009). Chondrocyte-specific Smad4 gene conditional knockout results in hearing loss and inner ear malformation in mice. Dev. Dyn. 238, 1897-1908. doi: 10.1002/ dvdy.22014

Yoon, H., Lee, D. J., Kim, M. H., and Bok, J. (2011). Identification of genes concordantly expressed with Atoh1 during inner ear development. Anat. Cell Biol. 44, 69-78. doi: 10.5115/acb.2011. 44.1.69

Zak, M., Bress, A., Pfister, M., and Blin, N. (2011). Temporal expression pattern of Fkbp8 in rodent cochlea. Cell. Physiol. Biochem. 28, 1023-1030. doi: 10.1159/ 000335789
Zhao, B., Wu, Z., Grillet, N., Yan, L., Xiong, W., Harkins-Perry, S., et al. (2014). TMIE is an essential component of the mechanotransduction machinery of cochlear hair cells. Neuron 84, 954-967. doi: 10.1016/j.neuron.2014.10.041

Conflict of Interest Statement: The authors declare that the research was conducted in the absence of any commercial or financial relationships that could be construed as a potential conflict of interest.

Copyright (c) 2017 Cheng, Guo, Lu, Xu, Zhang, Gao, Waqas, Zhu, Chen, Zhang, Xuan, Gao, Tang, Chen, Shi, Li and Chai. This is an open-access article distributed under the terms of the Creative Commons Attribution License (CC BY). The use, distribution or reproduction in other forums is permitted, provided the original author(s) or licensor are credited and that the original publication in this journal is cited, in accordance with accepted academic practice. No use, distribution or reproduction is permitted which does not comply with these terms. 